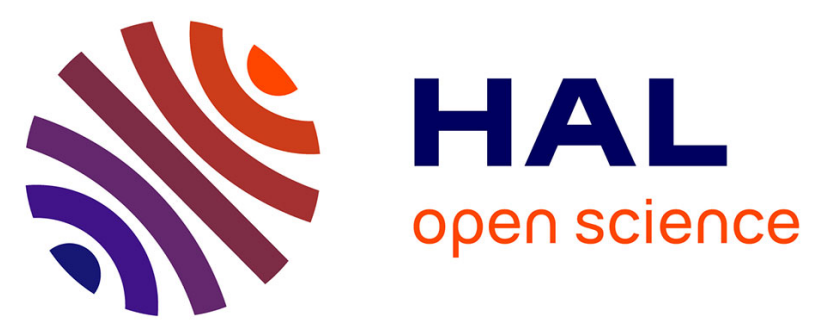

\title{
Unsteady compressible flow in ducts with varying cross-section: Comparison between the nonconservative Euler system and the axisymmetric flow model
}

David Rochette, Stéphane Clain, William Bussiere

\section{- To cite this version:}

David Rochette, Stéphane Clain, William Bussiere. Unsteady compressible flow in ducts with varying cross-section: Comparison between the nonconservative Euler system and the axisymmetric flow model. 2010. hal-00493771

\section{HAL Id: hal-00493771 \\ https://hal.science/hal-00493771}

Preprint submitted on 21 Jun 2010

HAL is a multi-disciplinary open access archive for the deposit and dissemination of scientific research documents, whether they are published or not. The documents may come from teaching and research institutions in France or abroad, or from public or private research centers.
L'archive ouverte pluridisciplinaire $\mathbf{H A L}$, est destinée au dépôt et à la diffusion de documents scientifiques de niveau recherche, publiés ou non, émanant des établissements d'enseignement et de recherche français ou étrangers, des laboratoires publics ou privés. 


\title{
Unsteady compressible flow in ducts with varying cross-section: Comparison between the nonconservative Euler system and the axisymmetric flow model
}

\author{
D. Rochette ${ }^{a}$, S. Clain ${ }^{b}, W$. Bussière $^{c}$ \\ ${ }^{a}$ Clermont Université, Université Blaise Pascal, LAEPT, BP 10448, F-63000 \\ CLERMONT-FERRAND. \\ ${ }^{\mathrm{b}}$ Institut de Mathématiques, CNRS UMR 5219, Université Paul Sabatier, F-31062 \\ TOULOUSE Cedex 4. \\ ${ }^{\mathrm{c}}$ Clermont Université, Université d'Auvergne, LAEPT, BP 10448, F-63000 \\ CLERMONT-FERRAND.
}

\begin{abstract}
Nonconservative hyperbolic system corresponds to a reduction of an initial threedimensional problem deriving from an homogenisation procedure. Unfortunately, the reduce model gives rise to two new difficulties : the resonant problem corresponding to a splitting or a merging of the genuinely nonlinear waves and the non uniqueness of the Riemann problem solution. The question arises to check whether the two problems correspond and provide similar solutions, at least numerically. In this paper, we propose a comparison between the one-dimensional nonconservative Euler equations modelling the duct with variable cross-sectional area with its original three-dimensional conservative Euler system. Based on the classification of the Riemann problems proposed in [11], we compare the numerical results of the two models for a large series of representative configurations. We also propose a new example of non uniqueness for the Riemann problem involving the resonant phenomena.
\end{abstract}

Key words: Riemann problem in ducts, nonconservative system, variable cross-section, axisymmetric flow, shock tube.

\section{Introduction}

Since two decades, nonconservative hyperbolic systems have received considerable attention both from a theoretical and a numerical points of view. Usually deriving from an homogenisation procedure of an initial three-dimensional 
model, several problems of physical or engineering importance are governed with a nonconservative hyperbolic system. We can mention the shallow-water problem with topography [15-17], the two-layer shallow-water problem [14], the sediment transport [8,28], the multi-fluid models [6], the Baer-Nunziato system [4], the compressible gas flow in variational cross-section duct [3] and the compressible gas flow in porous media [5,26,27]. For example, the nonconservative Euler equations, as well named the quasi-one-dimensional Euler in a duct, are obtained by averaging the usual multi-dimensional Euler equations over the duct cross-section [29].

Numerical approximations of the nonconservative hyperbolic system solutions have to be cleverly evaluated with specific numerical schemes named well-balanced schemes where the steady-state solutions are numerically preserved $[10,11,19,20]$. A large literature is now devoted on the subject [24,25]. The question we tackle in the article is the correspondence between the numerical solutions of the reduce problem, i.e. the nonconservative problem and the numerical solution computed with the initial problem i.e. the full three-dimensional one. Indeed, nonconservative systems give rise to essentially two new difficulties: the resonant phenomena and the non uniqueness of the solutions $[3,10,11,19]$ whereas such phenomena do not exist at the threedimensional level: the homogenisation procedure looses informations and the classical entropy argument (in the Lax sense for instance) is not enough to obtain a unique solution (numerically at least).

Few studies on the comparisons have been realised since such a problem has gained less attention and most of them only concern the shallow-water problem $[2,3]$. However the question seems of crucial importance for two reasons: firstly we have to check that the solutions of the two problems really correspond (at least numerically) for every type of configuration and secondly we have to select the most physical solution when we face a non uniqueness situation: we have several different entropy solutions (in the Lax sense) with the reduced model for the same Riemann problem and we choose the one which better fit with the three-dimensional solution.

To address the first issue, we deal with the variational cross-section duct problem or equivalently the gas flow in inhomogeneous porous media. We have implemented the scheme proposed in [11] to solve the nonconservative one-dimensional problem and the scheme proposed in [12] to solve the full three-dimensional axisymmetric one. We have numerically experimented all the available configurations proposed in [11] and compared it with their equivalent three-dimensional problem. We aim to prove that all the situations, even with the most complex ones like the splitting or merging of the simple genuinely nonlinear wave across the interface, are reproduced by the initial conservative Euler model which justify the reality of such configurations. The second issue we address concerns the non uniqueness of the solution for the reduced 
problem. A first example has been proposed by [3] where a subsonic solution and a supersonic solution are available for the same Riemann problem. We here propose a new non uniqueness case with a more complex situation: a supersonic solution and a resonant solution. Such an example proves that non uniqueness can also arise with complex configurations involving the merging or the splitting of the simple waves.

The organisation of the paper is as follows. In section 2, we present the governing Euler equations in cylindrical coordinates assuming rotational symmetry and the one-dimensional nonconservative Euler equations as well as a short review on the numerical techniques employed to discretize the equations. In section 3, we present all the admissible configurations for the Riemann problem in a duct with variable cross-section. Section 4 is devoted to the comparison between the exact solutions obtained with the inverse Riemann problem and the numerical approximations obtained by the one-dimensional nonconservative system and the axisymmetric flow model for all the configurations listed in [11]. In the section 5, we study the non uniqueness of the Riemann problem for the nonconservative system. We propose a new example involving a complex configuration with the resonant phenomenon. Finally, we propose in section 6 a set of simulations to compare the interaction of a planar shock wave entering in a duct with a rough or a smooth cross-section variation. Concluding remarks are made in section 7 .

\section{Gas flow models and numerical methods}

Perfect gas flow in variational cross-section is modelled with the three-dimensional axisymmetric Euler system where the cylindrical coordinates are employed to provide a simpler two-dimensional space variables problem with respect to the radial and axial coordinates $(r, x)$. Deriving from the primitive model, a nonconservative one-dimensional model - named the variational cross-section duct model $([3])$ - based on an homogenisation with respect to variable $r$ is obtained. The present section is dedicated to a short presentation of the two models and their respective numerical methods. Note that we use the $x$-axis as symmetry axis instead of the traditional $z$-axis for the three-dimensional model to provide a natural correspondence with the one-dimensional nonconservative model.

\subsection{Euler equations for axisymmetric flows}

Let us consider an axisymmetric domain $\Omega \times[0,2 \pi$ [ obtained by the rotation of a two-dimensional pattern $\Omega$ with respect to axis $\Delta$ corresponding to the 
azimuthal coordinate $x$. Remark that the domain is not cylindrical, a priori, since the orthogonal cross-section $S(x)$ may vary with $x$ as presented in figure 1.

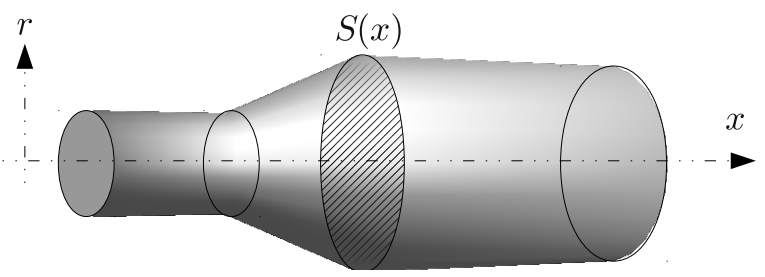

Fig. 1. Compressible fluid flow in axisymmetric geometry: the cross-section $S(x)$ is not constant leading to expansions or compressions of the gas when the section increases or decreases.

Using the cylindrical coordinate and assuming a non swirling flow, the Euler equations governing axially symmetric flow of a compressible inviscid perfect gas writes $[12,18,21]$ :

$$
\begin{aligned}
& \frac{\partial}{\partial t}(r \rho)+\frac{\partial}{\partial r}\left(r \rho u_{r}\right)+\frac{\partial}{\partial x}\left(r \rho u_{x}\right)=0, \\
& \frac{\partial}{\partial t}\left(r \rho u_{r}\right)+\frac{\partial}{\partial r}\left(r \rho u_{r}^{2}+r P\right)+\frac{\partial}{\partial x}\left(r \rho u_{r} u_{x}\right)=P, \\
& \frac{\partial}{\partial t}\left(r \rho u_{x}\right)+\frac{\partial}{\partial r}\left(r \rho u_{x} u_{r}\right)+\frac{\partial}{\partial x}\left(r \rho u_{x}^{2}+r P\right)=0, \\
& \frac{\partial}{\partial t}(r E)+\frac{\partial}{\partial r}\left(r u_{r}(E+P)\right)+\frac{\partial}{\partial x}\left(r u_{x}(E+P)\right)=0 .
\end{aligned}
$$

where $\rho, u_{r}, u_{x}$ and $E$ stand for the density, the radial and axial velocity and the total energy respectively. The pressure function $P$ is given by the perfect gas law depending on $\rho$ and $e$ the specific internal energy:

$$
P=(\gamma-1) \rho e \quad \text { and } \quad E=\rho e+\frac{1}{2} \rho\left(u_{r}^{2}+u_{x}^{2}\right),
$$

with $\gamma$ the ratio of specific heats.

The system cast under the generic expression:

$$
\frac{\partial(r U)}{\partial t}+\frac{\partial\left(r F_{r}(U)\right)}{\partial r}+\frac{\partial\left(r F_{x}(U)\right)}{\partial x}=G(U)
$$

where $U=\left(\rho, \rho u_{r}, \rho u_{x}, E\right)$ denotes the conservative variable vector, $F_{r}(U)=$ $\left(\rho u_{r}, \rho u_{r}^{2}+P, \rho u_{x} u_{r}, u_{r}(E+P)\right)$ and $F_{x}(U)=\left(\rho u_{x}, \rho u_{r} u_{x}, \rho u_{x}^{2}+P, u_{x}(E+P)\right)$ are the conservative fluxes and $G(U)=(0, P, 0,0)$ is the geometric source term deriving from the use of cylindrical coordinates. 
In the two last decades, several authors have developed numerical schemes for the Euler system in cylindrical coordinates (see [13,18,22,23] for instance). To address numerical approximation, we employ a second-order finite volume scheme proposed in [12] we shortly sum-up in the present article for the sake of consistency.

To this end, we consider a unstructured mesh $\mathcal{T}_{h}$ of the two-dimensional domain $\Omega$, constituted of triangles (or cells) $C_{i} \subset \Omega, i=1, \ldots, I$. We denote by $\nu(i)$ the index set of the neighbouring triangles $C_{j}$ which share a common edge $S_{i j}$ with the cell $C_{i}$ and by $\mathbf{n}_{i j}=\left(n_{i j, r}, n_{i j, x}\right)$ the outward unit normal vector to $C_{i}$.

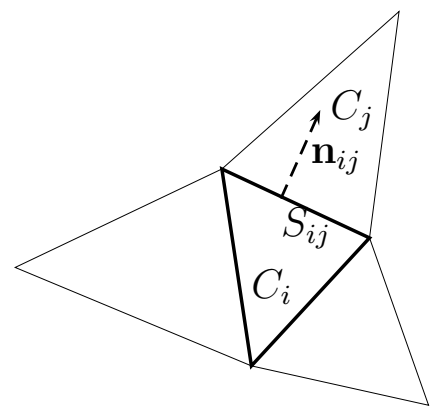

Fig. 2. The two-dimensional mesh: notations. Index set $\nu(i)$ represents the three neighbouring triangles of cell $C_{i}$.

Let $\left(t^{n}=n \Delta t\right)_{n \in \mathbb{N}}$ be a uniform subdivision of the time interval $[0,+\infty), U_{i}^{n}$ represents an approximation of the average of $U$ on $C_{i}$ at time $t^{n}$ :

$$
U_{i}^{n} \approx \frac{1}{\left|C_{i}\right|_{r}} \int_{C_{i}} U\left(r, x, t^{n}\right) r d r d x
$$

where we have introduced the weighted measures (cell and edge) :

$$
\left|C_{i}\right|=\int_{C_{i}} d r d x, \quad\left|C_{i}\right|_{r}=\int_{C_{i}} r d r d x, \quad\left|S_{i j}\right|_{r}=\int_{S_{i j}} r d \sigma
$$

In the same way, we define the approximation of the flux across the interface $S_{i j}$ during the time interval $\left[t^{n}, t^{n+1}\right]$ by

$$
F_{i j}^{n}=\mathcal{F}\left(U_{i}^{n}, U_{j}^{n}, \mathbf{n}_{i j}\right) \approx \frac{1}{\left|S_{i j}\right|_{r}} \int_{S_{i j}}\left(F_{r}(U) n_{i j, r}+F_{x}(U) n_{i j, x}\right) r d \sigma
$$


and the right-hand side contribution is approximated by

$$
G_{i}^{n}=G\left(U_{i}^{n}\right) \approx \frac{1}{\left|C_{i}\right|} \int_{C_{i}} G(U) d r d x
$$

A general finite volume scheme can be written as:

$$
\left|C_{i}\right|_{r} U_{i}^{n+1}=\left|C_{i}\right|_{r} U_{i}^{n}-\Delta t \sum_{j \in \nu(i)}\left|S_{i j}\right|_{r} \mathcal{F}\left(U_{i}^{n}, U_{j}^{n}, \mathbf{n}_{i j}\right)+\Delta t\left|C_{i}\right| G\left(U_{i}^{n}\right) .
$$

In the present study, we use the Rusanov flux combined with the multislope MUSCL technique on triangles developed in $[7,9]$ to reduce the diffusion effect (see also [12] for a detailed description). An important remark is that the scheme has been designed to respect a crucial well-balanced property: an initial state at rest remains a steady-state at rest.

\subsection{Nonconservative Euler system}

We now deal with the one-dimensional nonconservative Euler system describing the evolution of a perfect gas in a duct with variable cross-sectional area $S(x)$ following [3]. Let us denote by $\phi(x)=\frac{S(x)}{S_{r e f}}$ the ratio between the section area and a reference section, the equations then write:

$$
\begin{aligned}
& \frac{\partial}{\partial t}(\phi \rho)+\frac{\partial}{\partial x}(\phi \rho u)=0 \\
& \frac{\partial}{\partial t}(\phi \rho u)+\frac{\partial}{\partial x}\left(\phi \rho u^{2}+\phi P\right)=P \frac{\partial \phi}{\partial x}, \\
& \frac{\partial}{\partial t}(\phi E)+\frac{\partial}{\partial x}(\phi u(E+P))=0,
\end{aligned}
$$

and we assume that $\phi(x)$ only depends on $x$, i.e. $\frac{\partial \phi}{\partial t}=0$.

The system cast in the following nonconservative form:

$$
\frac{\partial U}{\partial t}+\frac{\partial F(U)}{\partial x}=G(U) \frac{\partial \phi}{\partial x}
$$

where conservative quantities are represented by vector $U=(\phi \rho, \phi \rho u, \phi E)$ and the flux vector by $F(U)=\left(\phi \rho u, \phi \rho u^{2}+\phi P, \phi u(E+P)\right)$ while the nonconservative term writes $G(U)=(0, P, 0)$.

To provide a numerical approximation of the solution of system (12), we first discretize domain $[a, b]$ with uniformly cells $C_{i}=\left[x_{i-1 / 2}, x_{i+1 / 2}\right], i=1, \ldots, I-1$ 
of length $\Delta x=\frac{b-a}{I}$ where we set $x_{i-1 / 2}=i \Delta x$ and $x_{i}=x_{i-1 / 2}+\frac{\Delta x}{2}$ is the cell center.

Like in the previous subsection, $U_{i}^{n}$ is an approximation of the mean value of $U$ on cell $C_{i}$ at time $t^{n}$ and we denote by $V_{i}^{n}$ the associated vector using the physical variables, $V=(\phi, \rho, u, P)$. We consider a generic well-balanced scheme of the form:

$$
U_{i}^{n+1}=U_{i}^{n}-\frac{\Delta t}{\Delta x}\left(F_{i+1 / 2}+G_{i+1 / 2}^{-}-F_{i-1 / 2}-G_{i-1 / 2}^{+}\right)
$$

where $F_{i+1 / 2}=\mathcal{F}\left(V_{i}, V_{i+1}\right)$ and $F_{i-1 / 2}=\mathcal{F}\left(V_{i-1}, V_{i}\right)$ represent the conservative numerical flux across interface $x_{i+1 / 2}$ and $x_{i-1 / 2}$ while $G_{i+1 / 2}^{-}=\mathcal{G}^{-}\left(V_{i}, V_{i+1}\right)$ and $G_{i-1 / 2}^{+}=\mathcal{G}^{+}\left(V_{i-1}, V_{i}\right)$ represent the nonconservative contribution across the interface consecutive to the $\phi$ space variation.

To compute the conservative fluxes we use the modified version of the Rusanov scheme

$$
\mathcal{F}\left(V_{i}, V_{i+1}\right)=\frac{F\left(V_{i}\right)+F\left(V_{i+1}\right)}{2}-\mathcal{V}\left(V_{i}, V_{i+1}\right),
$$

with

$$
\mathcal{V}\left(V_{i}, V_{i+1}\right)=\lambda \phi_{i+\frac{1}{2}}\left(\begin{array}{c}
\rho_{i+1}-\rho_{i} \\
\rho_{i+1} u_{i+1}-\rho_{i} u_{i} \\
E_{i+1}-E_{i}
\end{array}\right), \quad \phi_{i+\frac{1}{2}}=\max \left(\phi_{i}, \phi_{i+1}\right),
$$

whereas the nonconservative fluxes given by

$$
\mathcal{G}^{-}\left(V_{i}, V_{i+1}\right)=-\frac{\phi_{i+1}-\phi_{i}}{2}\left(\begin{array}{c}
0 \\
P_{i} \\
0
\end{array}\right), \quad \mathcal{G}^{+}\left(V_{i-1}, V_{i}\right)=+\frac{\phi_{i}-\phi_{i-1}}{2}\left(\begin{array}{c}
0 \\
P_{i} \\
0
\end{array}\right)
$$

are designed to satisfy the well-balanced property to preserve steady-states solutions (see [11] for a detailed construction of nonconservative schemes).

MUSCL reconstruction method is employed to provide a better accuracy of the numerical approximation and reduce the numerical diffusion. Due to the nonconservative term, one have to pay caution to design the higher-order scheme. We have developed a second-order reconstruction based on the splitting of the $\phi$ function into a regular part $\phi^{r}$ (say $C^{1}$ ) and a discontinuous piecewise 
constant one $\phi^{d}$

$$
\phi(x)=\phi^{d}(x)+\phi^{r}(x) .
$$

The main idea is that the regular part is treated as a classical volume source term whereas the discontinuous part is treated with the help of the nonconservative fluxes. We obtain a new generic finite volume scheme

$$
\begin{aligned}
U_{i}^{n+1}=U_{i}^{n}-\frac{\Delta t}{\Delta x}[ & \left\{\mathcal{F}\left(V_{i+1 / 2}^{n,-}, V_{i+1 / 2}^{n,+}\right)+\mathcal{G}^{-}\left(V_{i+1 / 2}^{n,-}, V_{i+1 / 2}^{n,+}\right)\right\}- \\
& \left.\left\{\mathcal{F}\left(V_{i-1 / 2}^{n,-}, V_{i-1 / 2}^{n,+}\right)+\mathcal{G}^{+}\left(V_{i-1 / 2}^{n,-}, V_{i-1 / 2}^{n,+}\right)\right\}+\mathcal{S}\left(V_{i+1 / 2}^{n,-}, V_{i-1 / 2}^{n,+}\right)\right]
\end{aligned}
$$

where the term $\mathcal{S}$ corresponds to the contribution of the regular part $P \frac{\partial \phi^{r}}{\partial x}$ given by

$$
\mathcal{S}\left(V_{i+1 / 2}^{n,-}, V_{i-1 / 2}^{n,+}\right)=\left(\begin{array}{c}
0 \\
\frac{P_{i-1 / 2}^{+}+P_{i+1 / 2}^{-}}{2}\left(\phi_{i+1 / 2}^{-}-\phi_{i-1 / 2}^{+}\right) \\
0
\end{array}\right) .
$$

\section{Riemann problem for the nonconservative Euler system}

The finite volume method is based on the solution of a local Riemann problem on every edges of the mesh. Therefore, from a computational point of view, the theoretical analysis of the Riemman problem is of crucial importance. A detailed study in the framework of the nonconservative Euler system has been carried out by Clain and Rochette in [11]. We recall here the main results.

The Riemann problem consists in solving the nonconservative hyperbolic system (12) on $\mathbb{R} \times \mathbb{R}^{+}$with the following initial condition:

$$
(U, \phi)(x, 0)=\left\{\begin{array}{l}
\left(U_{L}, \phi_{L}\right), x<0 \\
\left(U_{R}, \phi_{R}\right), x>0
\end{array}\right.
$$

where $\left(U_{L}, \phi_{L}\right)$ and $\left(U_{R}, \phi_{R}\right)$ are two given constant states.

The solution is constituted by a succession of simple waves (rarefaction, shock, contact discontinuity, stationary contact discontinuity) separated by constant states named configuration. The simple waves correspond to a change of state parameterised by the eigenvalues $\lambda_{0}=0, \lambda_{1}=u-c, \lambda_{2}=u$, and $\lambda_{3}=u+c$. 
The three last eigenvalues correspond to the classical Euler system while the first eigenvalue $\lambda_{0}$ characterises the brutal change of cross-section. We recall that the characteristic fields (or simple waves) associated to eigenvalues $\lambda_{1}$ and $\lambda_{3}$ are genuinely nonlinear providing rarefaction (noted $1-r$ or $3-r$ ) or shock (noted $1-s$ or $3-s$ ) while the characteristic fields associated to $\lambda_{0}$ and $\lambda_{2}$ are linearly degenerated (noted $0-w$ or $2-w$ ). We also say that a state $V=(\phi, \rho, u, P)$ is a subsonic, sonic or supersonic state whether we have $u^{2}<c^{2}, u^{2}=c^{2}$ or $u^{2}>c^{2}$ where $c$ stands for the sound velocity.

Since the system (12) is not strictly hyperbolic, simple waves can be distinct or superposed inducing a large number of configurations. To select the admissible configuration, we have introduced several criteria: the configuration stability, the sign and the Mach criteria (see $[11,19]$ ) leading to the following classification in four groups we shall precise in the next subsections:

(1) the classical wave configurations correspond to the situation where the four simple waves are separated by constant states (see figure 3).

(2) the splitting wave configurations correspond to a situation where a genuinely nonlinear wave is splitted by the $0-w$ wave (see figure 4 ).

(3) the resonant wave configurations correspond to the situation when a genuinely nonlinear wave splits the $0-w$ wave (see figure 5 ).

(4) the resonant and splitting wave configurations is the more complex case: a genuinely nonlinear wave is splitted by the $0-w$ wave where one of the part of the wave merges with the $0-w$ wave (see figure 6 ).

\subsection{Classical configurations}

The classical configurations represent the situations where the eigenvalues $\lambda_{k}$ are distinct corresponding to the Riemann problem for the conservative Euler system augmented of the stationary wave located at the interface $x=0$. We can list four distinct configurations in function of the simple waves position with respect to the interface $i . e$. the $0-w$ wave. If all the waves are on the left side (configuration $A$ ) or on the right side (configuration $D$ ) of the interface, we deal with a supersonic flow where all the states are supersonic. The two last configurations correspond to a subsonic flow whether the velocity at the interface is negative (configuration $B$ ) or positive (configuration $C$ ). As an example, figure 3 represents the $B$ configuration where the gas velocity at the interface is negative. Note that the $1-w$ simple wave is on the left while the $3-w$ simple wave is on the right. 


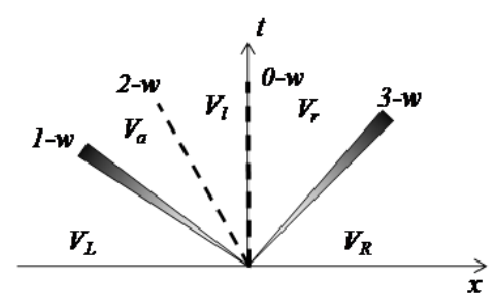

Fig. 3. Example of classical configurations: the $B$ configuration. The genuinely nonlinear simple waves $1-w$ and $3-w$ waves are rarefaction or shock waves whereas the $2-w$ wave is the $\lambda_{2}=u$ contact discontinuity and the $0-w$ wave is the stationary contact discontinuity wave corresponding to the cross-section discontinuity.

\subsection{Splitting configurations}

The configuration where one of the genuinely nonlinear $1-w$ or $3-w$ waves is splitted into two waves by the $0-w$ stationary wave on both side of the interface is a splitting configuration. It is proved that a rarefaction always takes place on the side corresponding to the lower $\phi_{L}$ or $\phi_{R}$. Moreover the fan must touch the interface which corresponds to a sonic state. Consequently, there is four distinct configurations in function of the values of $\phi$ and the genuinely nonlinear waves which are splitted. Configuration $L R_{1}$ (Left Rarefaction with the $1-w$ as shown in figure 4) corresponds to a rarefaction with a $1-w$ on the left side. Note that the second part of the $1-w$ is situated on the other side of the interface and can be an other rarefaction or a shock. In a similar way, we use the notations: $R R_{1}$ is a Right Rarefaction with the $1-w$ wave while $L R_{3}$ and $R R_{3}$ represent the Left Rarefaction and Right Rarefaction for the $3-w$ wave respectively.

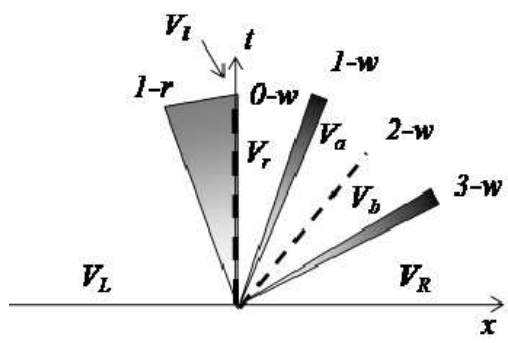

Fig. 4. Example of the splitting configurations: the $L R_{1}$ configuration. The $1-w$ is splitted into two parts, a rarefaction wave on the left side whom the fan reaches the interface and a rarefaction or a shock wave on the right side of the $0-w$ stationary wave due to the cross-section discontinuity. 


\subsection{Resonant wave configurations}

We now consider the symmetric case where a genuinely nonlinear $1-w$ or $3-w$ wave splits the stationary wave into two parts named the resonant configuration (see [19] p.892 case 1a (C) for example and [11]). For such a situation, the genuinely nonlinear wave is also a stationary shock corresponding to a subsonic-supersonic transition which takes place at an intermediate value of $\phi_{s} \in\left[\phi_{L}, \phi_{R}\right]$. These two situations are referenced by $R_{1}$ (see figure 5 for instance) or $R_{3}$ whether the resonant configuration is obtained with the first or the third eigenvalue.

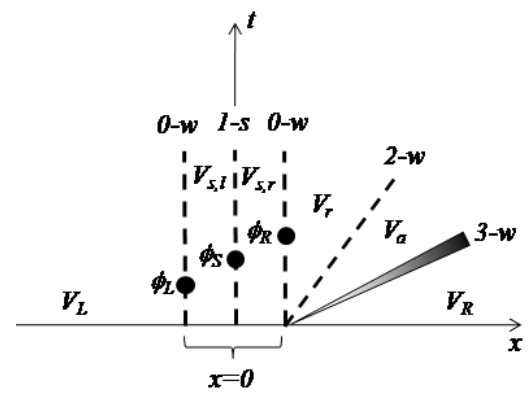

Fig. 5. Example of the resonant configurations: the $R_{1}$ configuration. The $1-s$ is a stationary shock wave superposed to the $0-w$ wave. From left to right, we have a first $0-w$ wave transition from $\phi_{L}$ to an intermediate value $\phi_{s}$. Then a stationary $1-s$ shock wave occurs followed by an other $0-w$ wave transition from $\phi_{s}$ to $\phi_{R}$. The two other $2-w$ and $3-w$ waves are the classical ones.

\subsection{Resonant and splitting wave configurations}

The last group is the most complicated one since we combine a resonant situation with a splitting situation. A genuinely nonlinear $1-w$ or $3-w$ wave is splitted into two pieces where the part situated on the lower cross-section is a rarefaction which touches the interface. The other part is a stationary shock sharing the $0-w$ wave in another two parts leading to a resonant situation. This configuration has been introduced in $[19,10,11]$. For example, as shown in figure 6 , the $1-w$ is splitted into a rarefaction on the left side of the interface. The first $0-w$ wave takes place corresponding to a transition from $\phi_{L}$ to the intermediate value $\phi_{s}$ followed by the second part of the stationary $1-s$ shock. At last, a second transition with the $0-w$ occurs from $\phi_{s}$ to $\phi_{R}$. The two other waves are the classical ones. The notation $L R R_{1}$ means that we deal with a Left Rarefaction and Resonant configuration with the $1-w$ simple wave while $R R R_{1}$ corresponds to the Right Rarefaction and Resonant configuration. In the same way, we have the $L R R_{3}$ and the $R R R_{3}$ configurations. 


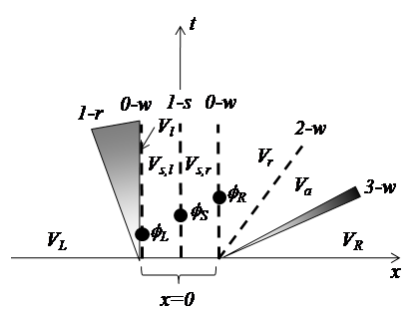

Fig. 6. Example of resonant splitting configurations: $L R R_{1}$ configuration. The first part of the $1-w$ wave is a $1-r$ rarefaction wave taking place on the left side of the interface and the second part is a $1-s$ stationary shock superposed to the $0-w$ stationary for an intermediate value $\phi_{s}$.

\section{Comparisons between the two models}

The aim of the current section is to draw comparisons between numerical solution of the Riemann problems presented in the previous section of the two models and the exact solution obtained with the inverse Riemann method (see [11]). A similar work has already been carried out by [3] using a twodimensional model to compare the solutions but only for the classical configurations. We propose here a systematic comparison of all the configurations. For the nonconservative Euler model, we consider a shock tube of length $x=\left[\begin{array}{ll}0 & : 2\end{array}\right]$ where the initial discontinuity is located at $x=0.8$ and we use a subdivision of 1000 mesh cells and an adapted time step to provide the stability. For the axisymmetric model, we consider a cylindrical shock tube of $x=[0: 2]$ length and the reference radius is arbitrarily chosen equal to $R_{\text {ref }}=0.05$ corresponding to $\phi=1$. Like in the previous case, the initial cross-section discontinuity is located at $x=0.8$. We use a 46000 triangle cells mesh and the time step is controlled to provide a stable solution. The two models are relied by the duct cross-section ratio with respect to the reference radius and we set :

$$
\phi_{L}=\frac{\pi R_{L}^{2}}{\pi R_{r e f}^{2}}, \quad \phi_{R}=\frac{\pi R_{R}^{2}}{\pi R_{r e f}^{2}},
$$

where $R_{L}$ and $R_{R}$ are respectively the cross-section duct radius on the left and right side of the interface.

We now give a detailed comparison of all the configurations. For a given left and right side, we report in the table the exact solutions (note that we can have several solutions) obtained by the inverse Riemann problem where we list the successive constant states from left to right. Then we print out the density and velocity of the gas for the one-dimensional nonconservative problem at a representative time $t_{r}$ and the graph of the same quantities in function of $x$ following the axis $r=0$ for the three-dimensional axisymmetric Euler case. We finally plot a cut of the plane Orx to provide the density map for the axisymmetric problem at the same time $t_{r}$. 


\subsection{Classical wave configurations}

\subsubsection{Configuration A}

Table 1

Configuration of type $A=\{1-r, 2-w, 3-s, 0-w\}$

\begin{tabular}{|c|c|c|c|c|c|}
\hline & $\phi$ & $\rho\left(\mathrm{kg} . \mathrm{m}^{-3}\right)$ & $\mathrm{u}\left({\left.\mathrm{m} . \mathrm{s}^{-1}\right)}\right.$ & $\mathrm{P}(\mathrm{Pa})$ & Mach \\
\hline \hline$V_{L}$ & 1.0 & 1 & -1000 & 400000 & 1.336306 \\
\hline$V_{a}$ & 1.0 & 0.714825 & -757.020985 & 250000 & 1.081867 \\
\hline$V_{b}$ & 1.0 & 0.5 & -757.020985 & 250000 & 0.904813 \\
\hline$V_{l}$ & 1.0 & 0.177419 & -1609.823851 & 50000 & 2.562889 \\
\hline$V_{R}$ & 0.5 & 0.432066 & -1322.085708 & 173835.409095 & 1.761576 \\
\hline
\end{tabular}
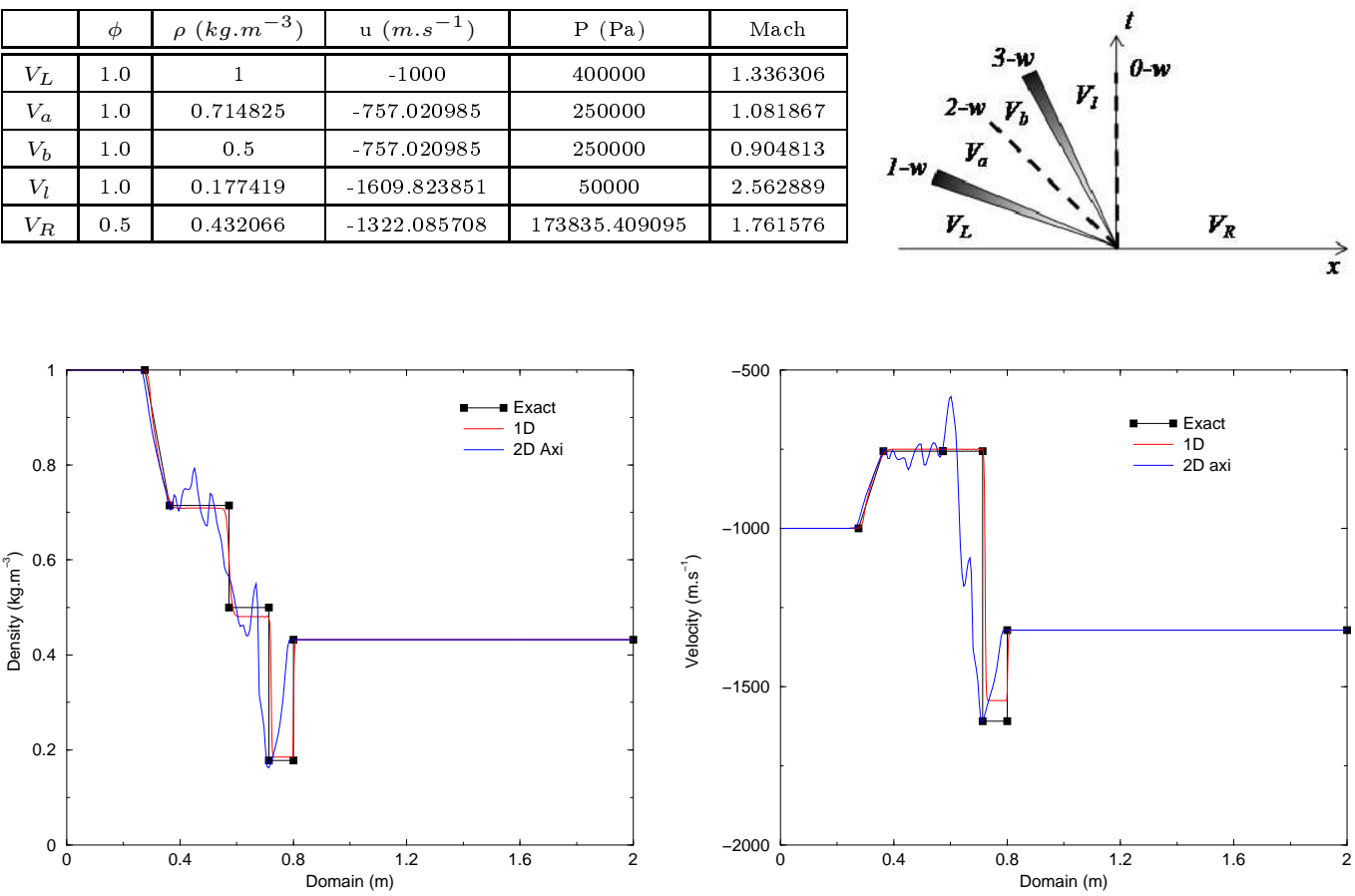

Fig. 7. Configuration of type $A=\{1-r, 2-w, 3-s, 0-w\}$ at time $3 \cdot 10^{-4} s$.

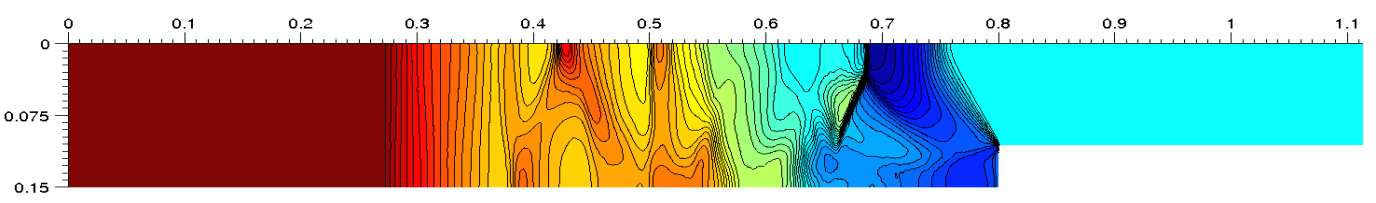

Fig. 8.50 isodensity from 1 to 0.09 at time $3 \cdot 10^{-4} s$.

Comments. Table 1 gives the exact solution obtained with the inverse Riemann problem while figures 7 and 8 show respectively the comparison between the exact solution and the numerical approximations for the density and velocity with the two schemes. We note that the 1D-solutions (exact or numerical) roughly correspond to the axisymmetric case. Indeed, the one-dimensional case corresponds to strict longitudinal waves following the axial direction while the numerical solution of the axisymmetric case provides oblique shocks leading to a more complex density distribution and a smoothing effect for the shocks. However, the axisymmetric numerical solution clearly catches the configuration $A$ in comparison with the exact solution. 


\subsubsection{Configuration B}

Table 2

Configuration of type $B=\{1-r, 2-w, 0-w, 3-s\}$

\begin{tabular}{|c|c|c|c|c|c|}
\hline & $\phi$ & $\rho\left(\mathrm{kg}^{\prime} \mathrm{m}^{-3}\right)$ & $\mathrm{u}\left(m \cdot s^{-1}\right)$ & $\mathrm{P}(\mathrm{Pa})$ & Mach \\
\hline$V_{L}$ & 1.0 & 3.6 & -100 & 400000 & 0.253546 \\
\hline$V_{a}$ & 1.0 & 2.931302 & -20.597552 & 300000 & 0.054415 \\
\hline$V_{l}$ & 1.0 & 4 & -20.597552 & 300000 & 0.063565 \\
\hline$V_{r}$ & 0.5 & 3.975401 & -41.450012 & 297420.273072 & 0.128075 \\
\hline$V_{R}$ & 0.5 & 1.597881 & -329.977019 & 75000 & 1.287245 \\
\hline
\end{tabular}
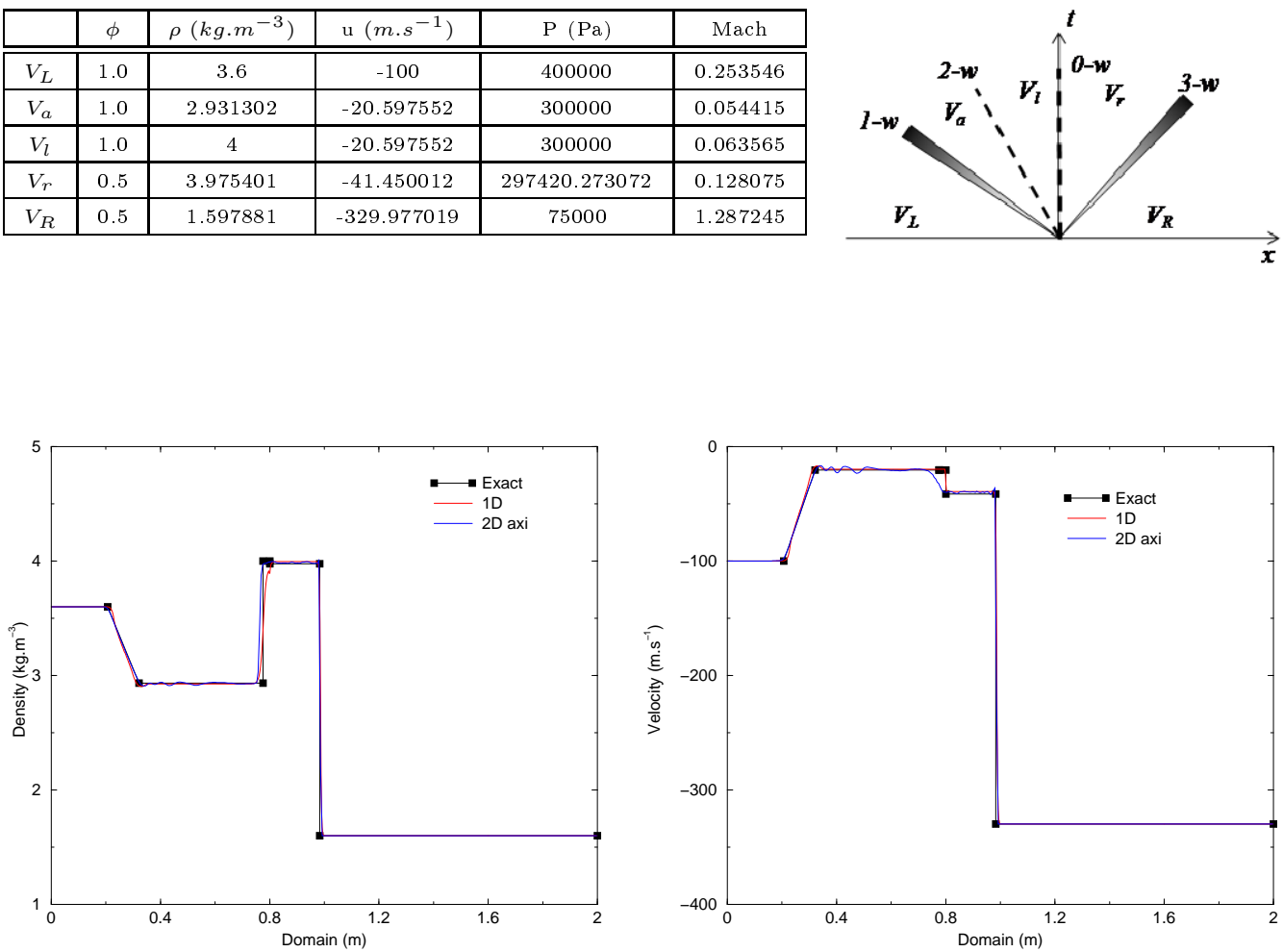

Fig. 9. Configuration of type $B=\{1-r, 2-w, 0-w, 3-s\}$ at time $12 \cdot 10^{-4} s$.

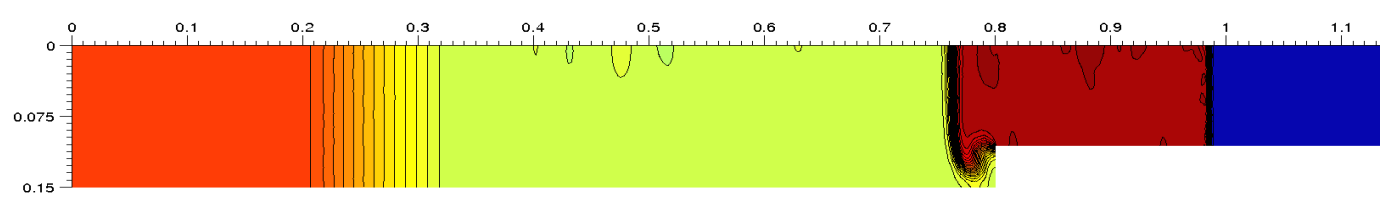

Fig. 10. 50 isodensity from 1.5 to 4 at time $12.10^{-4} \mathrm{~s}$.

Comments. Table 2 gives the exact solution obtained with the inverse Riemann problem while figures 9 and 10 show respectively the comparison between the exact solution and the numerical approximations for the density and velocity with the two schemes. For this configuration, we observe that the 1D-solutions (exact or numerical) perfectly fit with the axisymmetric solution since the shock waves are principally longitudinal reducing the smoothing effect. 


\subsubsection{Configuration $C$}

Table 3

Configuration of type $C=\{1-r, 0-w, 2-w, 3-s\}$

\begin{tabular}{|c|c|c|c|c|c|}
\hline & $\phi$ & $\rho\left(\mathrm{kg}^{\left.-\mathrm{m}^{-3}\right)}\right.$ & $\mathrm{u}\left(\mathrm{m.s} \mathrm{s}^{-1}\right)$ & $\mathrm{P}(\mathrm{Pa})$ & Mach \\
\hline \hline$V_{L}$ & 0.9 & 3.6 & 100 & 300000 & 0.292770 \\
\hline$V_{l}$ & 0.9 & 2.694778 & 196.112884 & 200000 & 0.608399 \\
\hline$V_{r}$ & 1.0 & 2.828882 & 168.134511 & 214071.314011 & 0.516560 \\
\hline$V_{a}$ & 1.0 & 3.4 & 168.134511 & 214071.314011 & 0.566308 \\
\hline$V_{R}$ & 1.0 & 3.238852 & 153.784754 & 200000 & 0.523034 \\
\hline
\end{tabular}
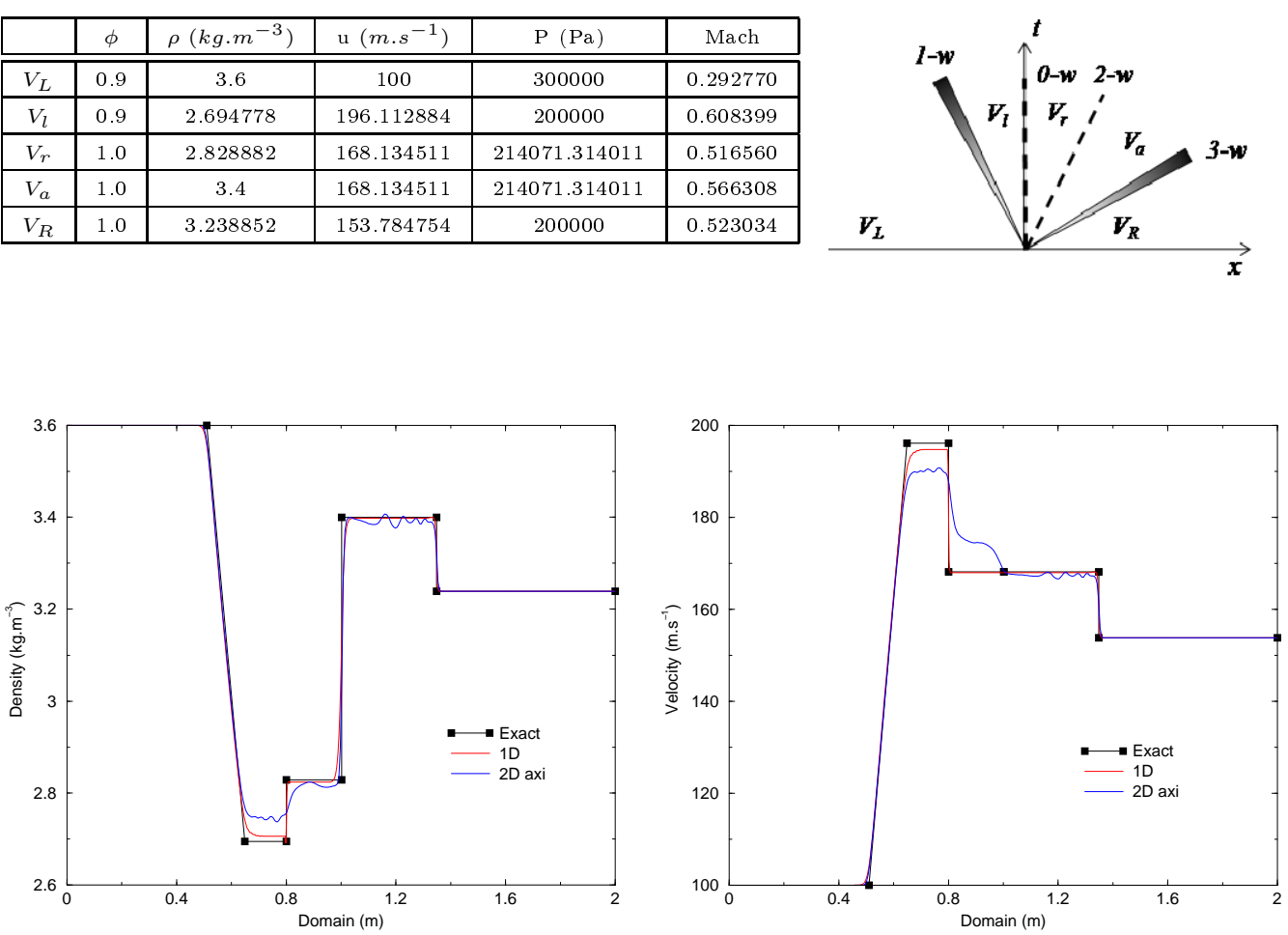

Fig. 11. Configuration of type $C=\{1-r, 0-w, 2-w, 3-s\}$ at time $12 \cdot 10^{-4} s$.

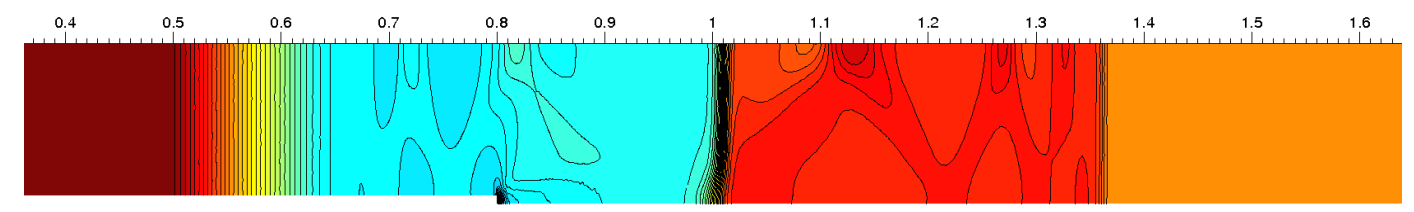

Fig. 12.50 isodensity from 2.3 to 3.6 at time $12.10^{-4} \mathrm{~s}$.

Comments. Table 3 gives the exact solution obtained with the inverse Riemann problem while figures 11 and 12 show respectively the comparison between the exact solution and the numerical approximations for the density and velocity with the two schemes. In the vicinity of the cross-section discontinuity, we note differences between the solutions of the axisymmetric problem and the nonconservative one. The very small jump of cross-section seems to be smoothed in the three-dimensional context while the one-dimensional model preserves the stationary shock. However, the other waves fit well with the exact solution. 


\subsubsection{Configuration D}

Table 4

Configuration of type $D=\{0-w, 1-s, 2-w, 3-s\}$

\begin{tabular}{|c|c|c|c|c|c|}
\hline & $\phi$ & $\rho\left(k g . \mathrm{m}^{-3}\right)$ & $\mathrm{u}\left({\left.\mathrm{m} . \mathrm{s}^{-1}\right)}\right.$ & $\mathrm{P}(\mathrm{Pa})$ & Mach \\
\hline \hline$V_{L}$ & 0.7 & 1 & 800 & 80000 & 2.390457 \\
\hline$V_{r}$ & 1.0 & 0.656948 & 852.426066 & 44425.652469 & 2.770393 \\
\hline$V_{a}$ & 1.0 & 1.154958 & 661.437525 & 100000 & 1.899800 \\
\hline$V_{b}$ & 1.0 & 4 & 661.437525 & 100000 & 3.535532 \\
\hline$V_{R}$ & 1.0 & 3.259259 & 623.748607 & 75000 & 3.475157 \\
\hline
\end{tabular}
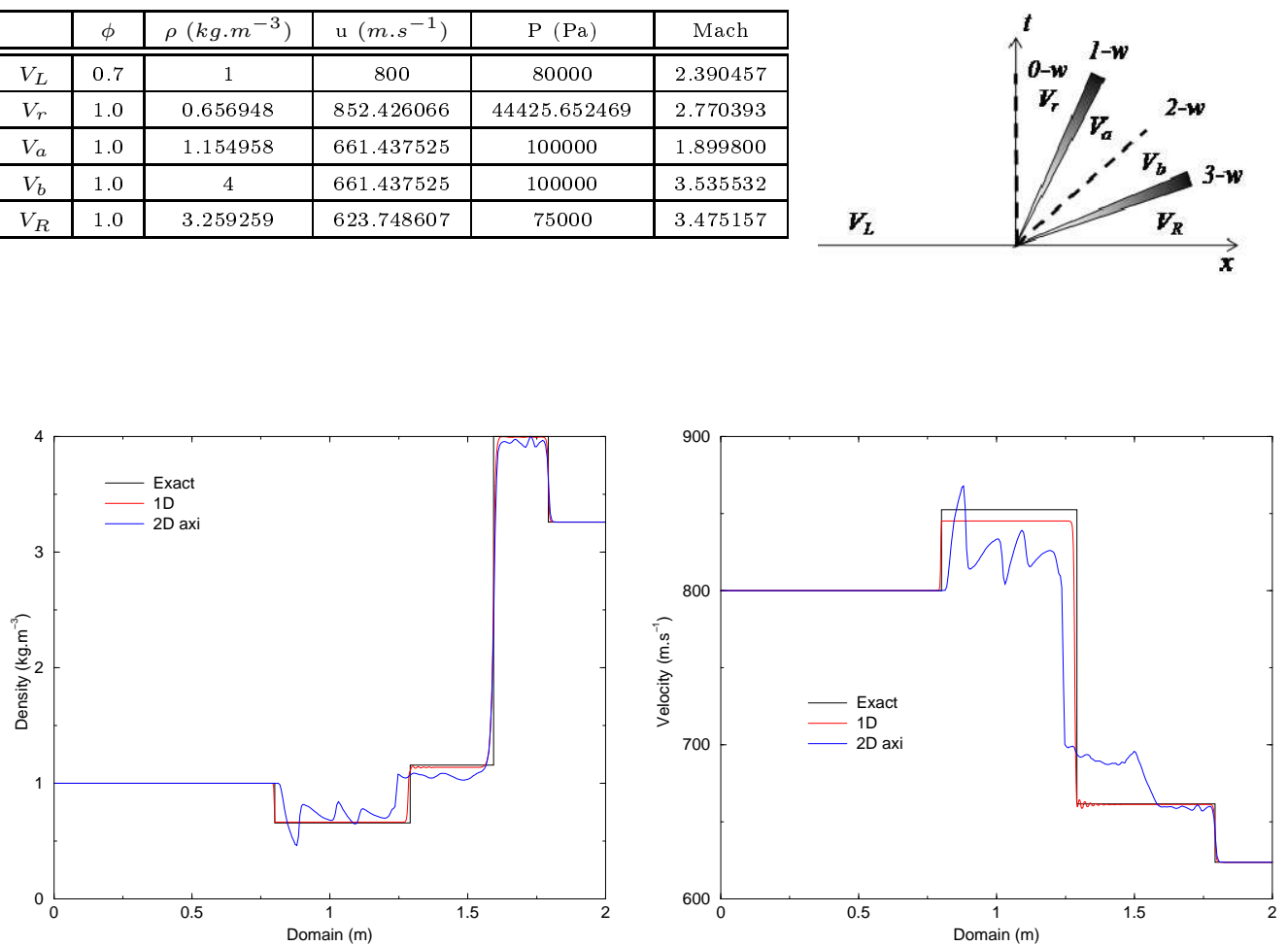

Fig. 13. Configuration of type $D=\{0-w, 1-s, 2-w, 3-s\}$ at time $12 \cdot 10^{-4} s$.

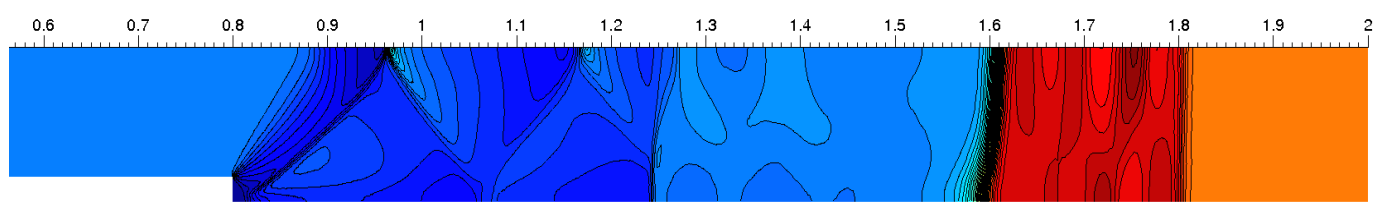

Fig. 14. 50 isodensity from 0.06 to 4.3 at time $12.10^{-4} \mathrm{~s}$.

Comments. Table 4 gives the exact solution obtained with the inverse Riemann problem while figures 13 and 14 show respectively the comparison between the exact solution and the numerical approximations for the density and velocity with the two schemes. The profiles are roughly respected in the shock tube but we note important oscillations of the axisymmetric solution (essentially on the first left plateau). Like configuration $A$, we observe slanted waves stemming from the three-dimensional context (radial waves are generated at the interface). 


\subsection{Splitting wave configurations}

\subsubsection{Configuration $L R_{1}$ with rarefaction}

Table 5

Configuration of type $L R_{1}$ with rarefaction $L R_{1} \_r a r=\{1-r, 0-w, 1-r, 2-w, 3-r\}$

\begin{tabular}{|c|c|c|c|c|c|}
\hline & $\phi$ & $\rho\left(k g . m^{-3}\right)$ & $\mathrm{u}\left(\mathrm{m.s}^{-1}\right)$ & $\mathrm{P}(\mathrm{Pa})$ & Mach \\
\hline \hline$V_{L}$ & 0.8 & 5 & 250 & 400000 & 0.747018 \\
\hline$V_{l}$ & 0.8 & 4.031127 & 320.553342 & 295868.736587 & 1.0 \\
\hline$V_{r}$ & 1.0 & 2.262737 & 456.859511 & 131822.563570 & 1.599708 \\
\hline$V_{a}$ & 1.0 & 1.583820 & 555.189919 & 80000 & 2.087783 \\
\hline$V_{b}$ & 1.0 & 1.68 & 555.189919 & 80000 & 2.150241 \\
\hline$V_{R}$ & 1.0 & 2.376396 & 647.909308 & 130000 & 2.341197 \\
\hline
\end{tabular}
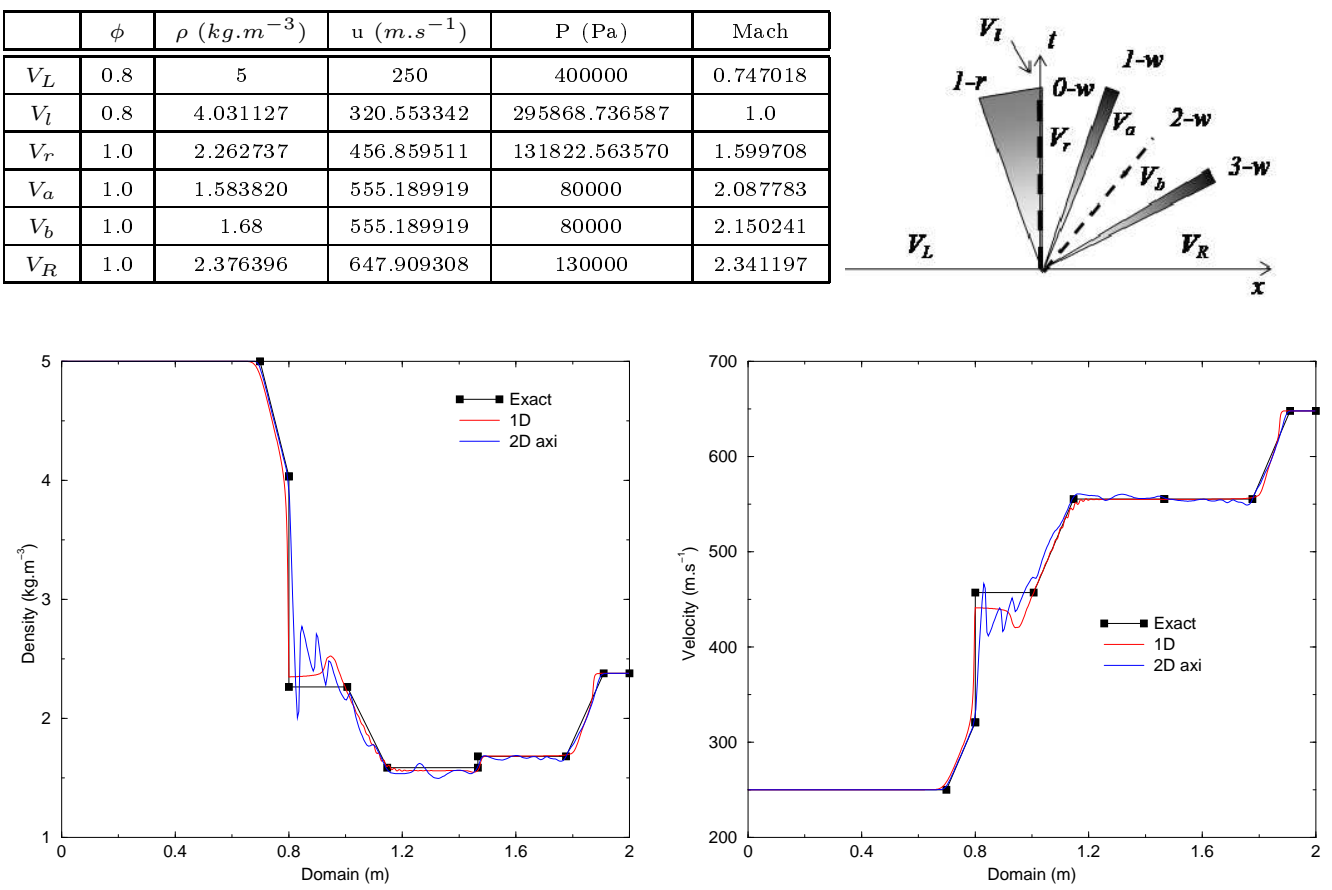

Fig. 15. Configuration of type $L R_{1} \_r a r=\{1-r, 0-w, 1-r, 2-w, 3-r\}$ at time $12.10^{-4} \mathrm{~s}$.

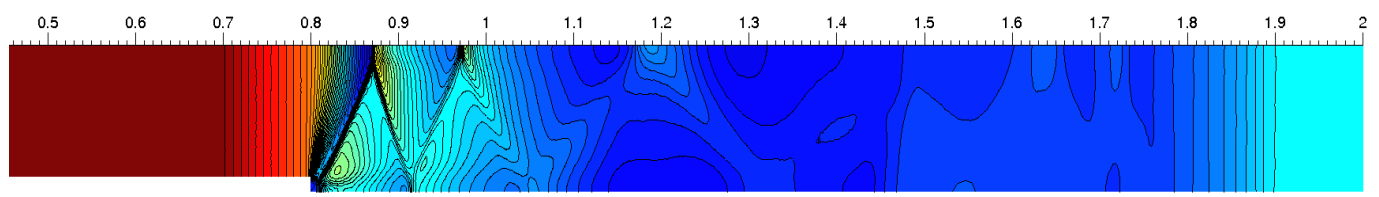

Fig. 16. 50 isodensity from 1 to 5 at time $12 \cdot 10^{-4} \mathrm{~s}$.

Comments. Table 5 gives the exact solution obtained with the inverse Riemann problem while figures 15 and 16 show respectively the comparison between the exact solution and the numerical approximations for the density and velocity with the two schemes. We first remark that both the 1D-model and the 3D-model present left rarefaction which spreads out from the left subsonic state so far to a sonic state at the interface as stated by the theory in [11]. The curves fit well despite some oscillations of the $V_{r}$ state for the three-dimensional configuration consecutive to oblique shocks. Thanks to our experience and after a lot of numerical experiments, it appears that this particular state (just before or just after a rarefaction reaching the sonic point) is always difficult to accurately approximate. It is one of the most sensitive state. 


\subsubsection{Configuration $L R_{1}$ with shock}

Table 6

Configuration of type $L R_{1}$ with shock $L R_{1} \_s c k=\{1-r, 0-w, 1-s, 2-w, 3-s\}$

\begin{tabular}{|c|c|c|c|c|c|}
\hline & $\phi$ & $\rho\left(k g . m^{-3}\right)$ & $\mathrm{u}\left({\mathrm{m} . \mathrm{s}^{-1}}^{-1}\right.$ & $\mathrm{P}(\mathrm{Pa})$ & Mach \\
\hline \hline$V_{L}$ & 0.8 & 5 & 200 & 300000 & 0.690066 \\
\hline$V_{l}$ & 0.8 & 3.835307 & 274.856279 & 206958.559906 & 1.0 \\
\hline$V_{r}$ & 1.0 & 2.152820 & 391.731075 & 92209.160840 & 1.599708 \\
\hline$V_{a}$ & 1.0 & 3.037373 & 303.313516 & 150000 & 1.153536 \\
\hline$V_{b}$ & 1.0 & 1.68 & 303.313516 & 150000 & 0.857900 \\
\hline$V_{R}$ & 1.0 & 1.033846 & 136.275239 & 75000 & 0.427612 \\
\hline
\end{tabular}
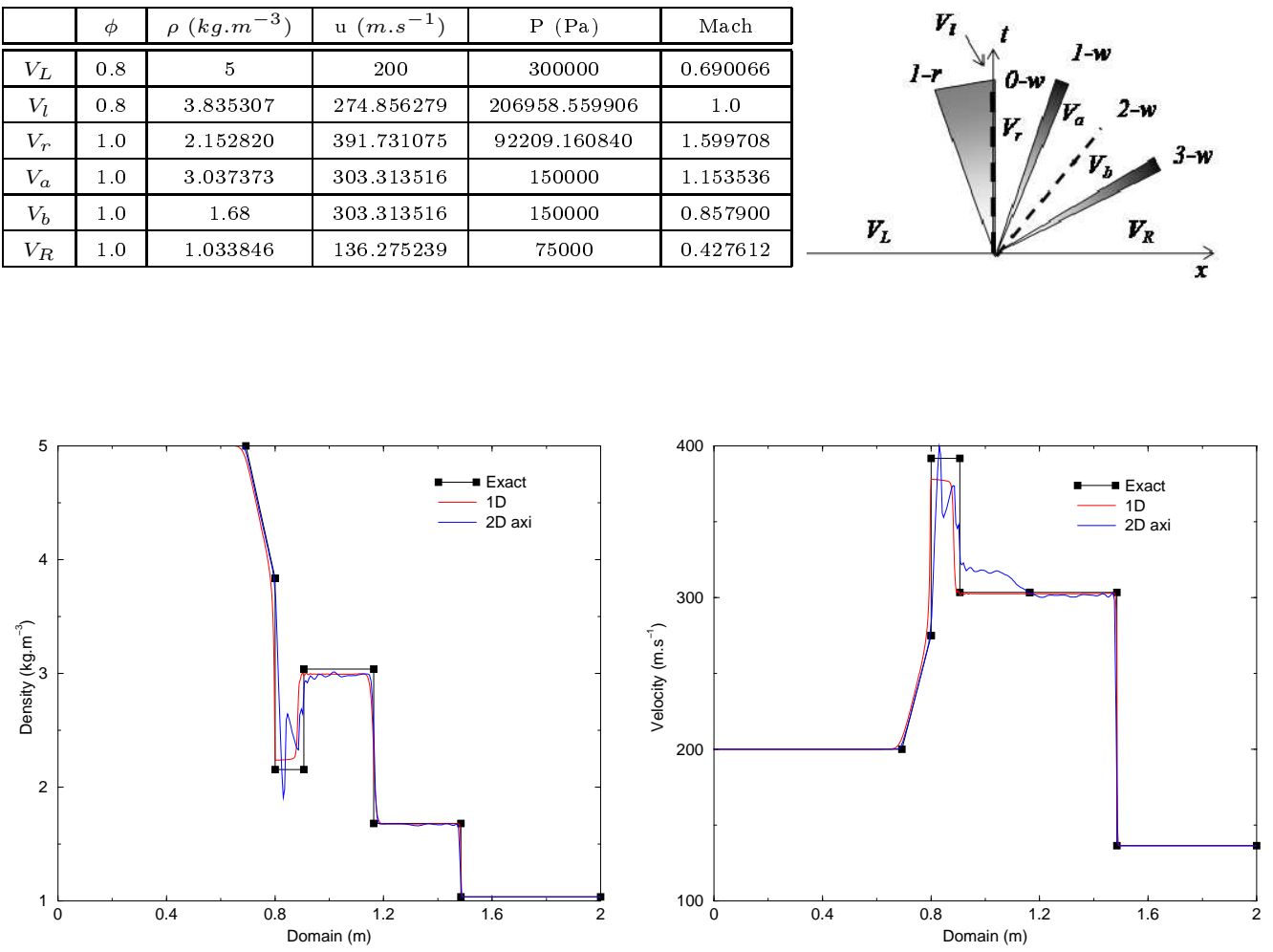

Fig. 17. Configuration of type $L R_{1} \_s c k=\{1-r, 0-w, 1-s, 2-w, 3-s\}$ at time $12.10^{-4} \mathrm{~s}$.

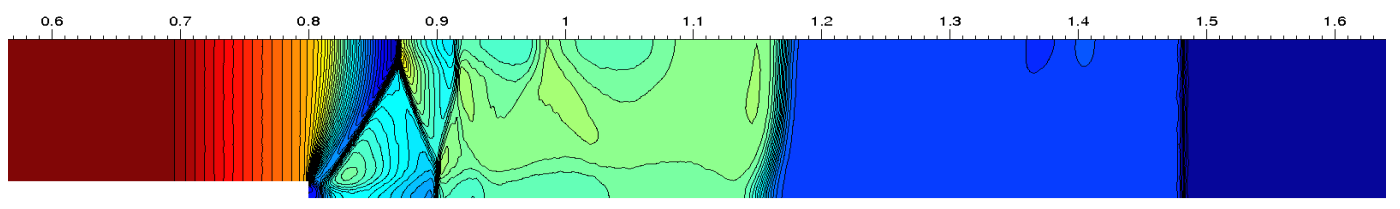

Fig. 18. 50 isodensity from 1 to 5 at time $12 \cdot 10^{-4} s$.

Comments. Table 6 gives the exact solution obtained with the inverse Riemann problem while figures 17 and 18 show respectively the comparison between the exact solution and the numerical approximations for the density and velocity with the two schemes. The previous comments apply to this configuration: the intermediate state $V_{r}$ for the $3 \mathrm{D}$-solution presents strong oscillations and we observe that the $1-s$ shock computed with the 1D-model parting the $V_{r}$ and $V_{a}$ states is smoothed. However, the axisymmetric solution clearly coincides well with the one-dimensional solutions. 


\subsubsection{Configuration $\mathrm{LR}_{3}$ with rarefaction}

Table 7

Configuration of type $L R_{3}$ with rarefaction $L R_{3}$ rar $=\{1-s, 2-w, 3-r, 0-w, 3-r\}$

\begin{tabular}{|c|c|c|c|c|c|}
\hline & $\phi$ & $\rho\left(k g . \mathrm{m}^{-3}\right)$ & $\mathrm{u}\left(\mathrm{m} . \mathrm{s}^{-1}\right)$ & $\mathrm{P}(\mathrm{Pa})$ & Mach \\
\hline \hline$V_{L}$ & 0.5 & 2 & -200 & 400000 & 0.377964 \\
\hline$V_{a}$ & 0.5 & 2.666667 & -358.113883 & 600000 & 0.638066 \\
\hline$V_{b}$ & 0.5 & 10.0 & -358.113883 & 600000 & 1.235610 \\
\hline$V_{l}$ & 0.5 & 12.123795 & -301.208593 & 785679.190499 & 1.0 \\
\hline$V_{r}$ & 1.0 & 18.258264 & -100.003790 & 1393780.920329 & 0.305904 \\
\hline$V_{R}$ & 1.0 & 26.112017 & 21.244529 & 2300000.0 & 0.060498 \\
\hline
\end{tabular}
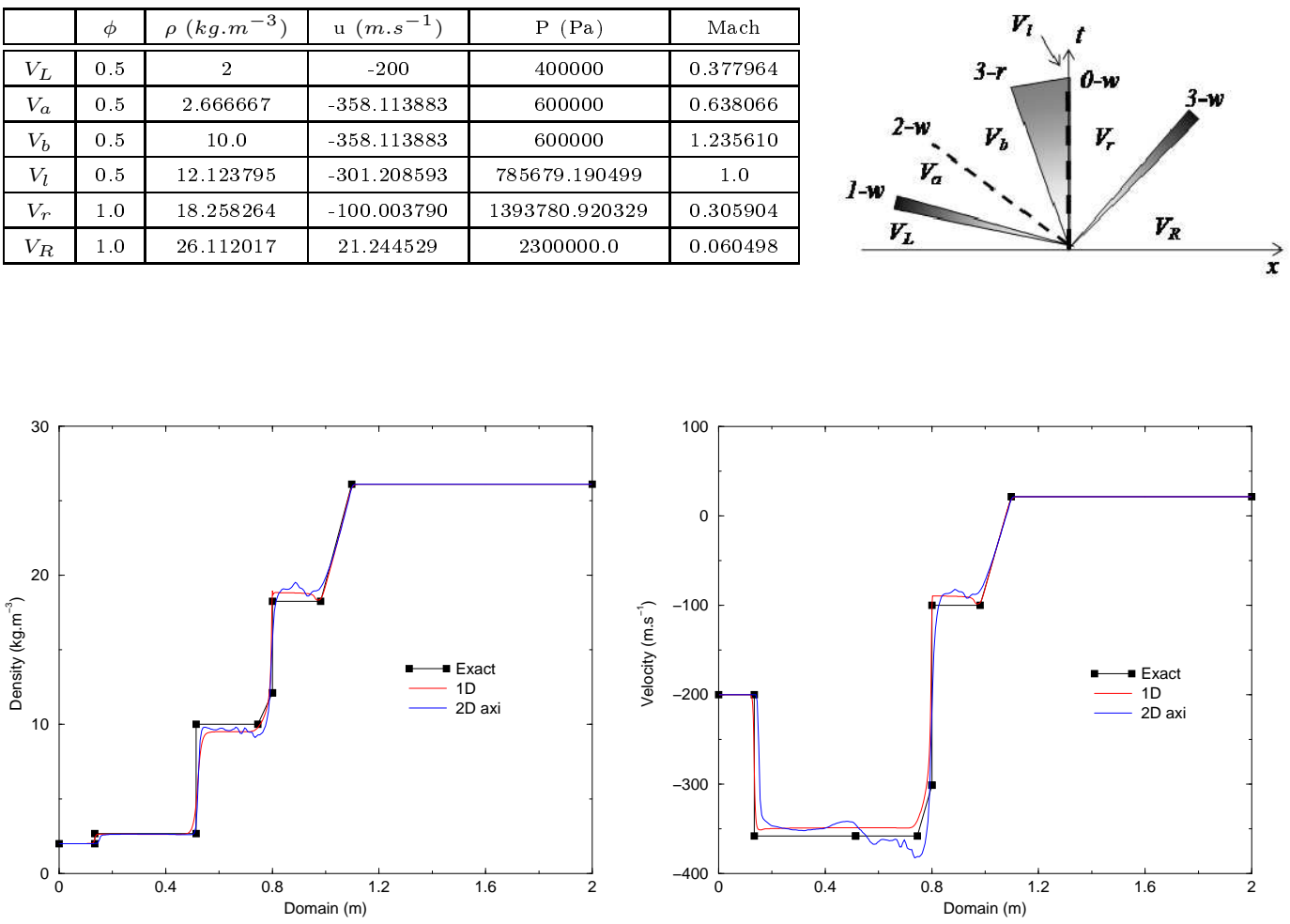

Fig. 19. Configuration of type $L R_{3}$ rar $=\{1-s, 2-w, 3-r, 0-w, 3-r\}$ at time $8.10^{-4} \mathrm{~s}$.

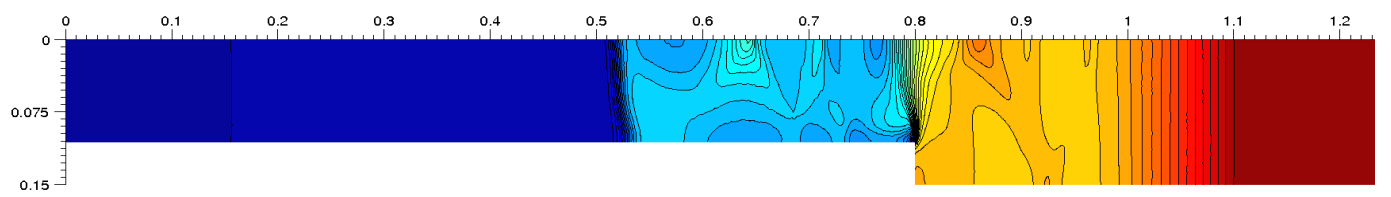

Fig. 20. 50 isodensity from 2 to 27 at time $8 \cdot 10^{-4} s$.

Comments. Table 7 gives the exact solution obtained with the inverse Riemann problem while figures 19 and 20 show respectively the comparison between the exact solution and the numerical approximations for the density and velocity with the two schemes. The theoretical small $3-r$ rarefaction which takes place from state $V_{b}$ to the sonic point $V_{l}$ predicted by the onedimensional model is not well reproduced by the three-dimensional model. One more time, small oscillations consecutive to oblique waves before and after the cross-section change appear. Nevertheless, the intermediate states correspond and the three simulations provide a $L R_{3}$ configuration. 


\subsubsection{Configuration $L R_{3}$ with shock}

Table 8

Configuration of type $L R_{3}$ with shock $L R_{3} \_s c k=\{1-s, 2-w, 3-r, 0-w, 3-s\}$

\begin{tabular}{|c|c|c|c|c|c|}
\hline & $\phi$ & $\rho\left(k g . \mathrm{m}^{-3}\right)$ & $\mathrm{u}\left(\mathrm{m} . \mathrm{s}^{-1}\right)$ & $\mathrm{P}(\mathrm{Pa})$ & Mach \\
\hline \hline$V_{L}$ & 0.5 & 5 & -250 & 400000 & 0.747018 \\
\hline$V_{a}$ & 0.5 & 6.666667 & -350 & 600000 & 0.986013 \\
\hline$V_{b}$ & 0.5 & 10.0 & -350 & 600000 & 1.207615 \\
\hline$V_{l}$ & 0.5 & 11.854071 & -299.856279 & 761317.446137 & 1.0 \\
\hline$V_{r}$ & 1.0 & 17.852064 & -99.554810 & 1350563.618802 & 0.305904 \\
\hline$V_{R}$ & 1.0 & 4.619603 & -542.096262 & 130000.0 & 2.731134 \\
\hline
\end{tabular}
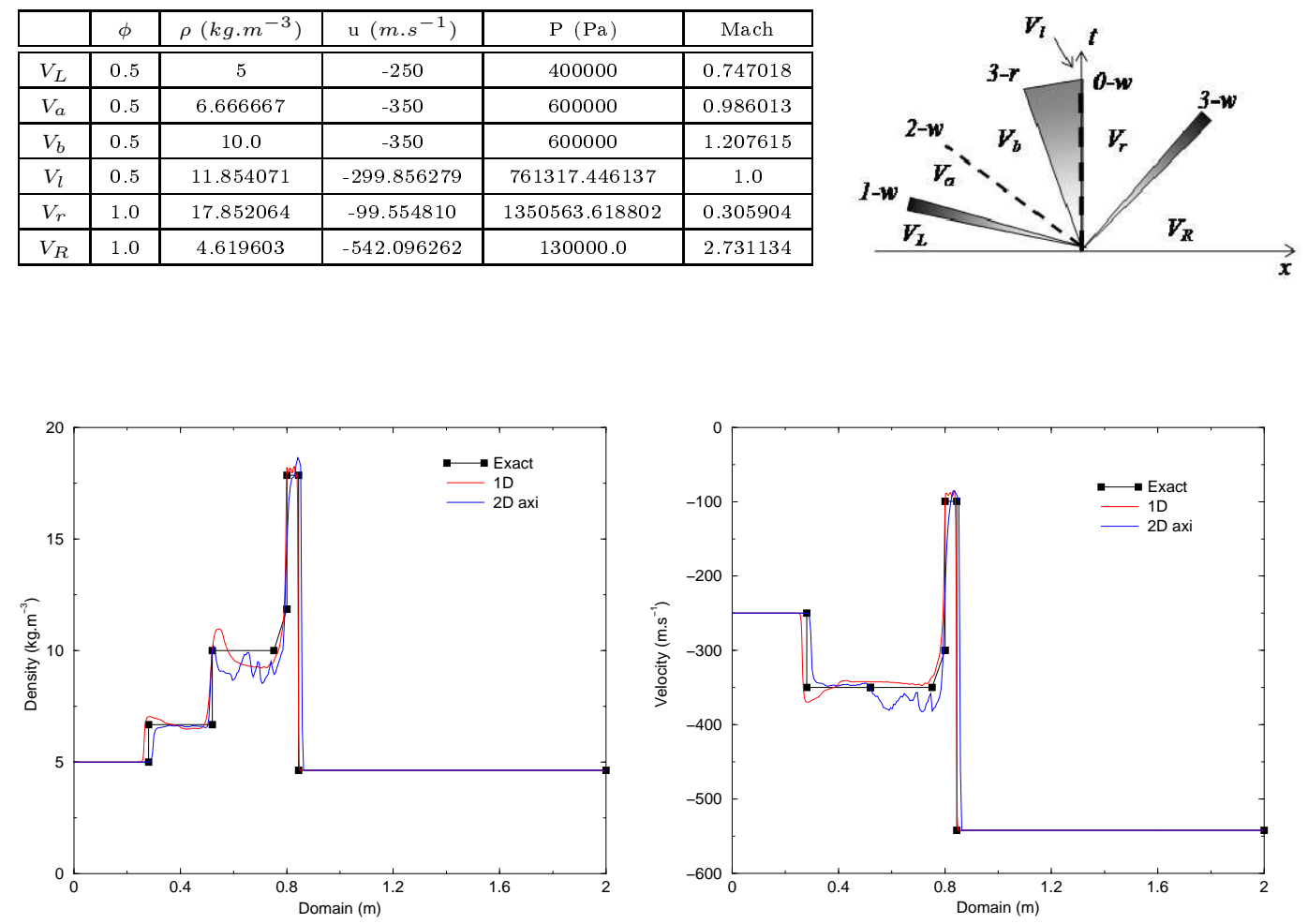

Fig. 21. Configuration of type $L R_{3} \_s c k=\{1-s, 2-w, 3-r, 0-w, 3-s\}$ at time $8.10^{-4} \mathrm{~s}$.

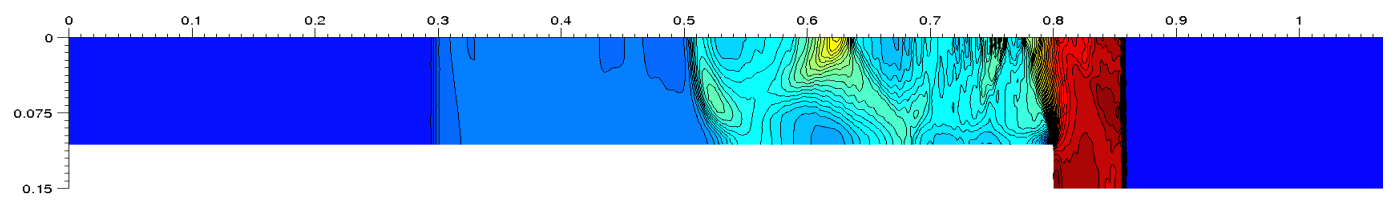

Fig. 22. 50 isodensity from 3 to 19 at time $8 \cdot 10^{-4} s$.

Comments. Table 8 gives the exact solution obtained with the inverse Riemann problem while figures 21 and 22 show respectively the comparison between the exact solution and the numerical approximations for the density and velocity with the two schemes. The present simulation is very similar to the former one but we have, in this case, a $3-s$ shock to link $V_{r}$ to $V_{R}$ on the right side of the interface while we have a $3-r$ rarefaction to link the states in the previous case. Comments and remarks are very similar, the three simulations provide the same configuration but oscillations due to oblique shocks appear with the three-dimensional model. 


\subsubsection{Configuration $R R_{1}$ with rarefaction}

Table 9

Configuration of type $R R_{1}$ with rarefaction $R R_{1} \_r a r=\{1-r, 0-w, 1-r, 2-w, 3-s\}$

\begin{tabular}{|c|c|c|c|c|c|}
\hline & $\phi$ & $\rho\left(k g . m^{-3}\right)$ & $\mathrm{u}\left({\left.\mathrm{m} . \mathrm{s}^{-1}\right)}\right.$ & $\mathrm{P}(\mathrm{Pa})$ & Mach \\
\hline \hline$V_{L}$ & 1.0 & 3.6 & 0.0 & 400000 & 0.0 \\
\hline$V_{l}$ & 1.0 & 2.501047 & 138.544575 & 240218.917576 & 0.377818 \\
\hline$V_{r}$ & 0.6 & 1.701108 & 339.491071 & 140042.899031 & 0.999999 \\
\hline$V_{a}$ & 0.6 & 1.337395 & 419.224375 & 100000 & 1.295723 \\
\hline$V_{b}$ & 0.6 & 0.7 & 419.224375 & 100000 & 0.937414 \\
\hline$V_{R}$ & 0.6 & 0.570370 & 329.130629 & 75000 & 0.767100 \\
\hline
\end{tabular}
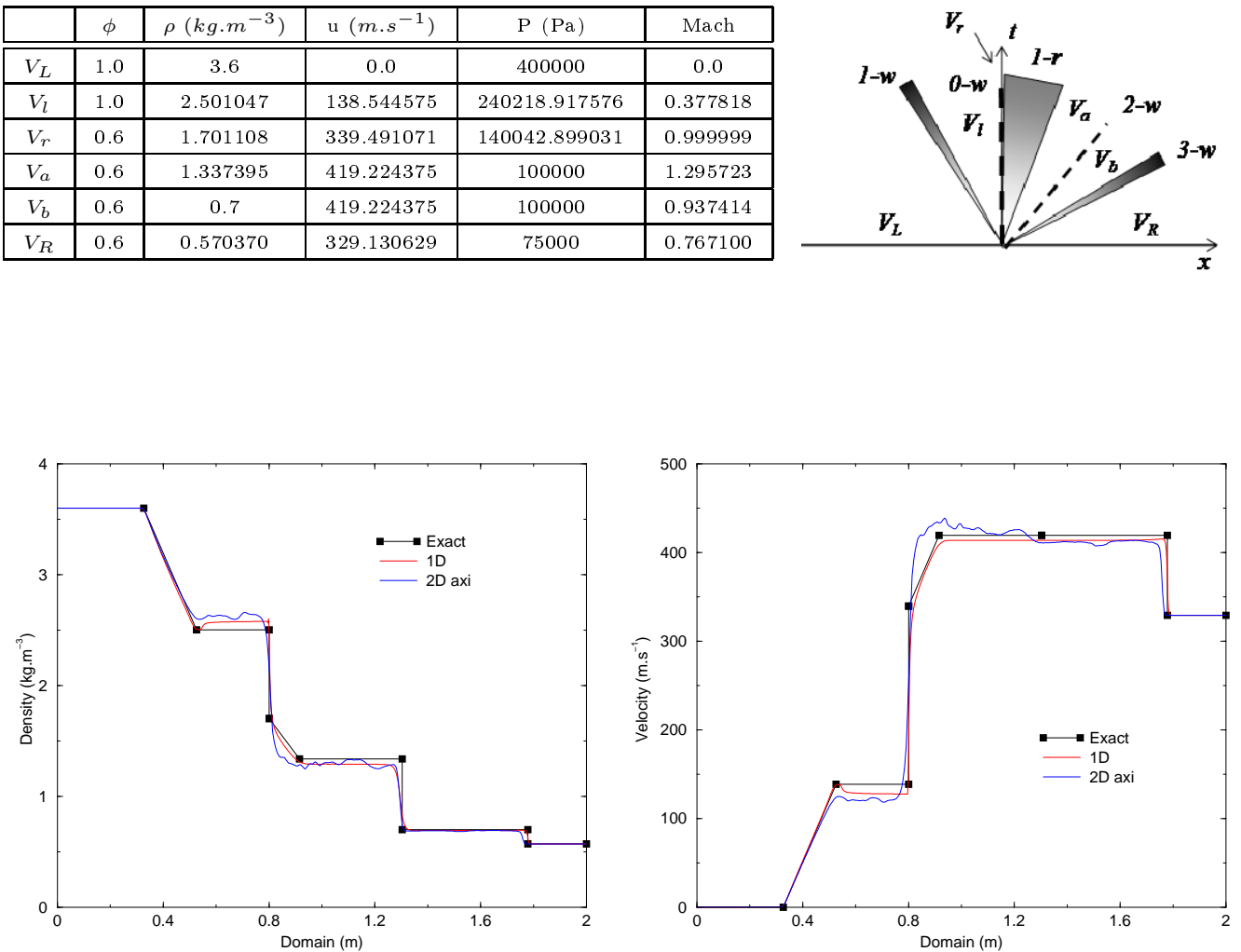

Fig. 23. Configuration of type $R R_{1}$ rar $=\{1-r, 0-w, 1-r, 2-w, 3-s\}$ at time $12.10^{-4} \mathrm{~s}$.

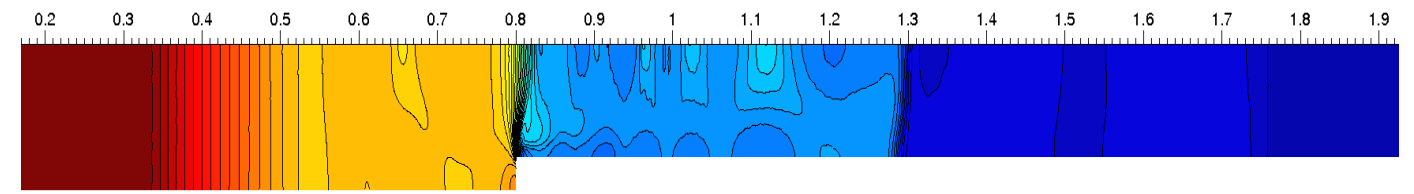

Fig. 24. 50 isodensity from 0.5 to 3.6 at time $12.10^{-4} \mathrm{~s}$.

Comments. Table 9 gives the exact solution obtained with the inverse Riemann problem while figures 23 and 24 show respectively the comparison between the exact solution and the numerical approximations for the density and velocity with the two schemes. We now consider situations when the lowest cross-section is on the right side leading this time to a right rarefaction. We observe that for this particular configuration the three curves suit well. 


\subsubsection{Configuration $R R_{1}$ with shock}

Table 10

Configuration of type $R R_{1}$ with shock $R R_{1} \_s c k=\{1-s, 0-w, 1-r, 2-w, 3-s\}$

\begin{tabular}{|c|c|c|c|c|c|}
\hline & $\phi$ & $\rho\left(k g . \mathrm{m}^{-3}\right)$ & $\mathrm{u}\left({\left.\mathrm{m} . \mathrm{s}^{-1}\right)}\right.$ & $\mathrm{P}(\mathrm{Pa})$ & Mach \\
\hline \hline$V_{L}$ & 1.0 & 3 & 100 & 200000 & 0.327327 \\
\hline$V_{l}$ & 1.0 & 3.259671 & 74.409093 & 224662.868292 & 0.239543 \\
\hline$V_{r}$ & 0.4 & 2.126227 & 285.187189 & 123521.258857 & 1.0 \\
\hline$V_{a}$ & 0.4 & 1.559044 & 370.984366 & 80000.0 & 1.384127 \\
\hline$V_{b}$ & 0.4 & 1.0 & 370.984366 & 80000.0 & 1.108528 \\
\hline$V_{R}$ & 0.4 & 0.862385 & 322.059761 & 65000.0 & 0.991440 \\
\hline
\end{tabular}
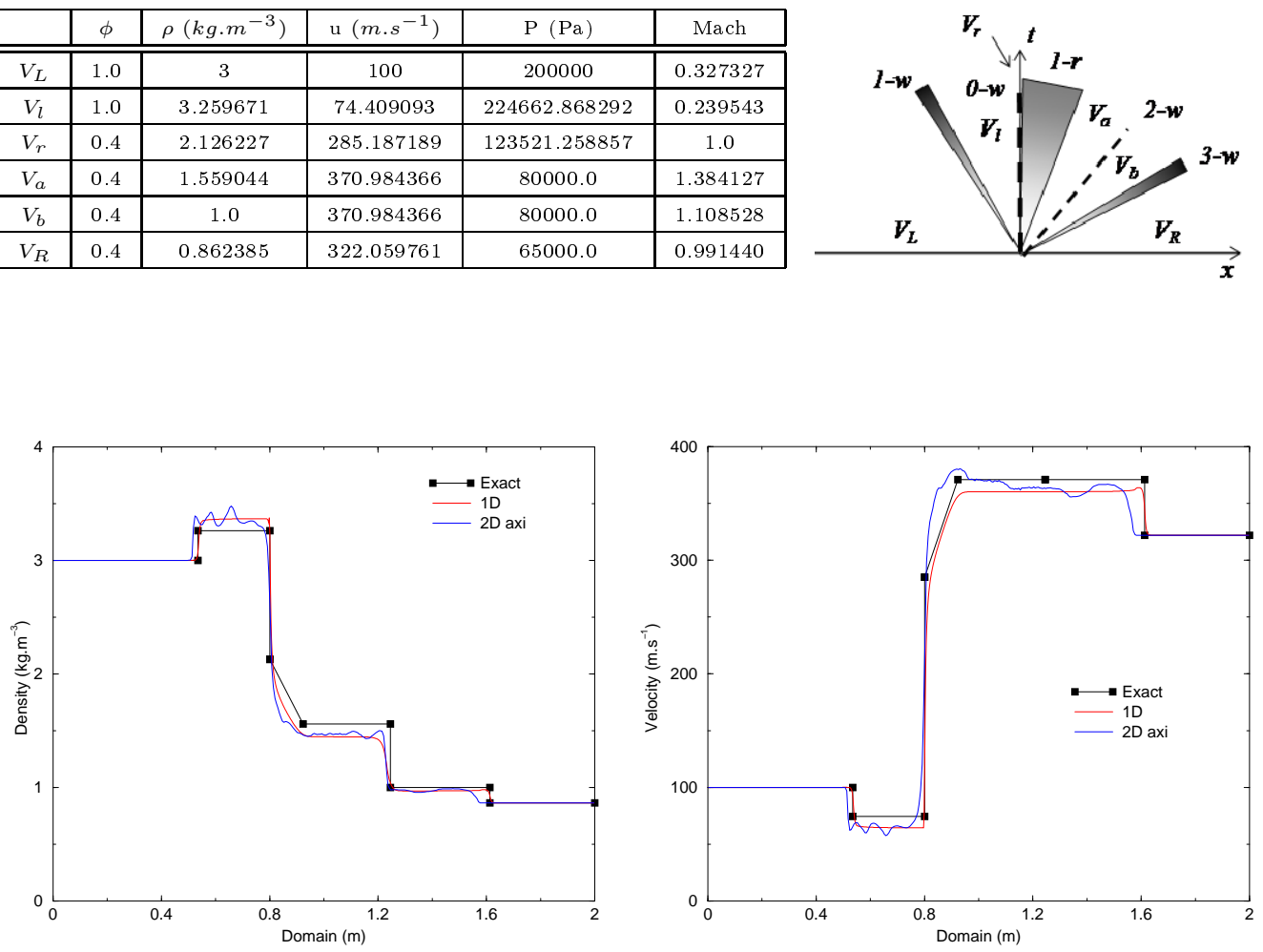

Fig. 25. Configuration of type $R R_{1} \_s c k=\{1-s, 0-w, 1-r, 2-w, 3-s\}$ at time $12.10^{-4} \mathrm{~s}$.

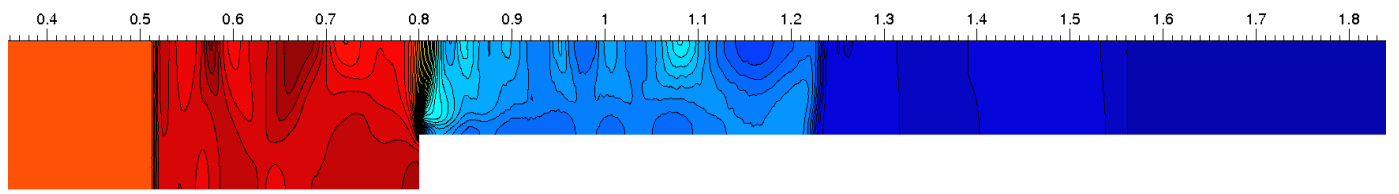

Fig. 26. 50 isodensity from 0.8 to 3.6 at time $12.10^{-4} \mathrm{~s}$.

Comments. Table 10 gives the exact solution obtained with the inverse Riemann problem while figures 25 and 26 show respectively the comparison between the exact solution and the numerical approximations for the density and velocity with the two schemes. In contrast with the previous case, the second part of the $1-w$ wave which shares the states $V_{L}$ and $V_{l}$ is a shock (a rarefaction in the former case). Note that despite the large cross-section ratio, we obtain the same configuration between the "real" three-dimensional model and the "homogenised" one-dimensional model. 


\subsubsection{Configuration $R R_{3}$ with rarefaction}

Table 11

Configuration of type $R R_{3}$ with rarefaction $R R_{3}$ r rar $=\{1-s, 2-w, 3-r, 0-w, 3-r\}$

\begin{tabular}{|c|c|c|c|c|c|}
\hline & $\phi$ & $\rho\left(k g . m^{-3}\right)$ & $\mathrm{u}\left({\mathrm{m} . \mathrm{s}^{-1}}^{-1}\right.$ & $\mathrm{P}(\mathrm{Pa})$ & Mach \\
\hline \hline$V_{L}$ & 1.0 & 5 & -250 & 100000 & 1.494036 \\
\hline$V_{a}$ & 1.0 & 6.666667 & -300 & 150000 & 1.690309 \\
\hline$V_{b}$ & 1.0 & 8 & -300 & 150000 & 1.851640 \\
\hline$V_{l}$ & 1.0 & 9.648037 & -269.076202 & 194975.414173 & 1.599708 \\
\hline$V_{r}$ & 0.8 & 17.188238 & -188.796060 & 437611.952725 & 1.0 \\
\hline$V_{R}$ & 0.8 & 22.801474 & 133.905733 & 650000 & 0.670286 \\
\hline
\end{tabular}
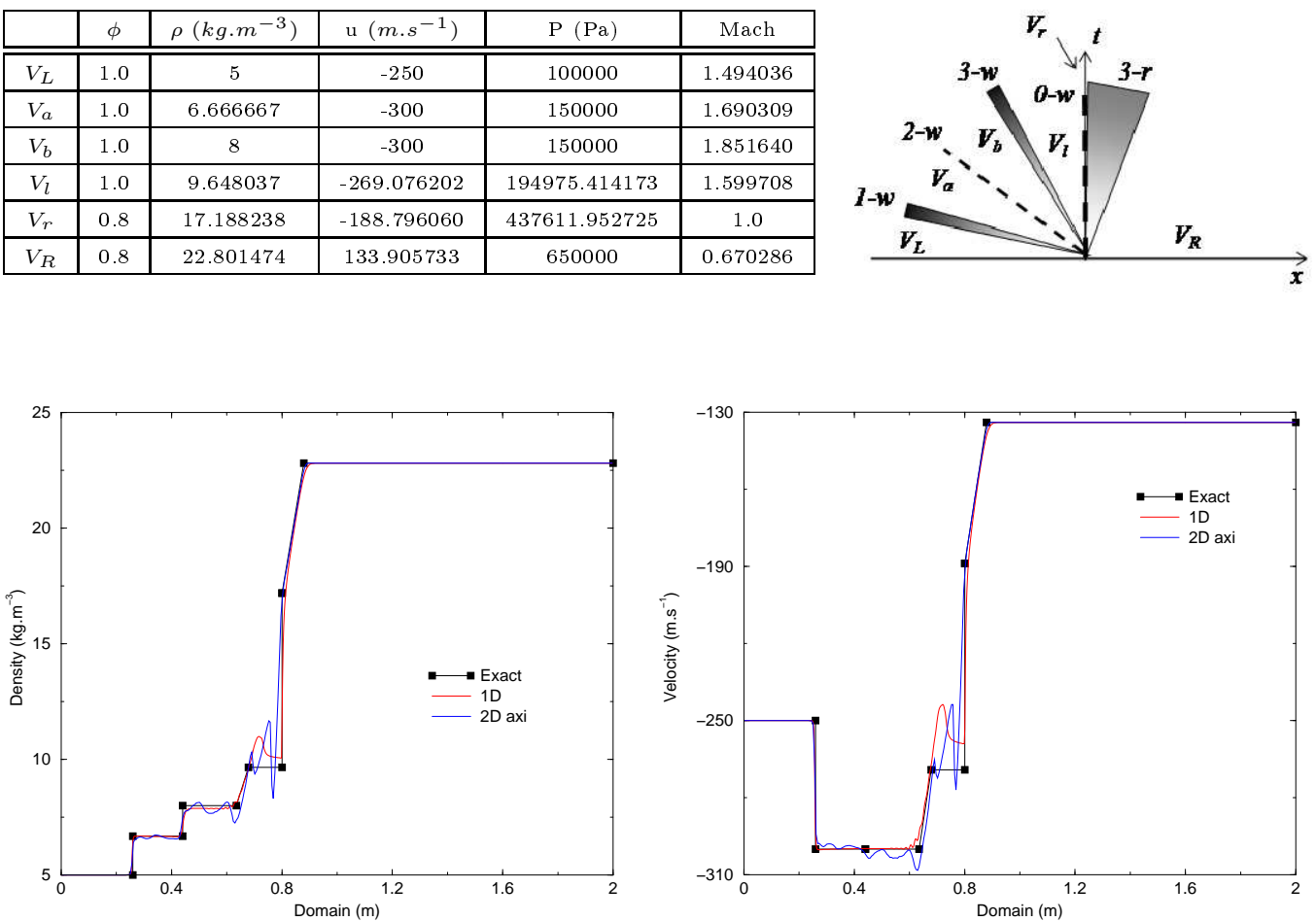

Fig. 27. Configuration of type $R R_{3}$ rar $=\{1-s, 2-w, 3-r, 0-w, 3-r\}$ at time $12.10^{-4} \mathrm{~s}$.

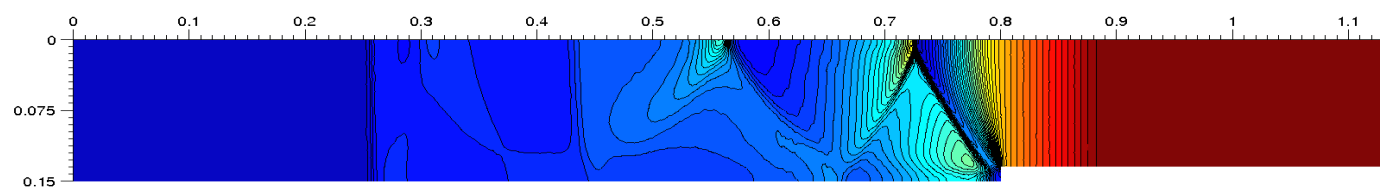

Fig. 28. 50 isodensity from 4 to 23 at time $12 \cdot 10^{-4} s$.

Comments. Table 11 gives the exact solution obtained with the inverse Riemann problem while figures 27 and 28 show respectively the comparison between the exact solution and the numerical approximations for the density and velocity with the two schemes. The present simulation shows clearly the oscillations origin. Strong slanted shocks propagate and the nice plateau (constant solution) obtained with the one-dimensional is poorly reproduced by the axisymmetric solution. Nevertheless, the theoretical configuration predicted by the 1D-model is confirmed by the 3D-simulation which indicates the adequacy of the homogenised model with the "real" one. 


\subsubsection{Configuration $R R_{3}$ with shock}

Table 12

Configuration of type $R R_{3}$ with shock $R R_{3} \_s c k=\{1-s, 2-w, 3-s, 0-w, 3-r\}$

\begin{tabular}{|c|c|c|c|c|c|}
\hline & $\phi$ & $\rho\left(k g . m^{-3}\right)$ & $\mathrm{u}\left({\mathrm{m} . \mathrm{s}^{-1}}^{-1}\right.$ & $\mathrm{P}(\mathrm{Pa})$ & Mach \\
\hline \hline$V_{L}$ & 1.0 & 3 & -400 & 50000 & 2.618615 \\
\hline$V_{a}$ & 1.0 & 4.875 & -480.064077 & 100000.0 & 2.832843 \\
\hline$V_{b}$ & 1.0 & 1.0 & -480.064077 & 100000.0 & 1.283025 \\
\hline$V_{l}$ & 1.0 & 0.783632 & -569.600910 & 70964.939235 & 1.599708 \\
\hline$V_{r}$ & 0.8 & 1.396062 & -399.657818 & 159277.033801 & 1.0 \\
\hline$V_{R}$ & 0.8 & 1.642596 & -333.595729 & 200000.0 & 0.807992 \\
\hline
\end{tabular}
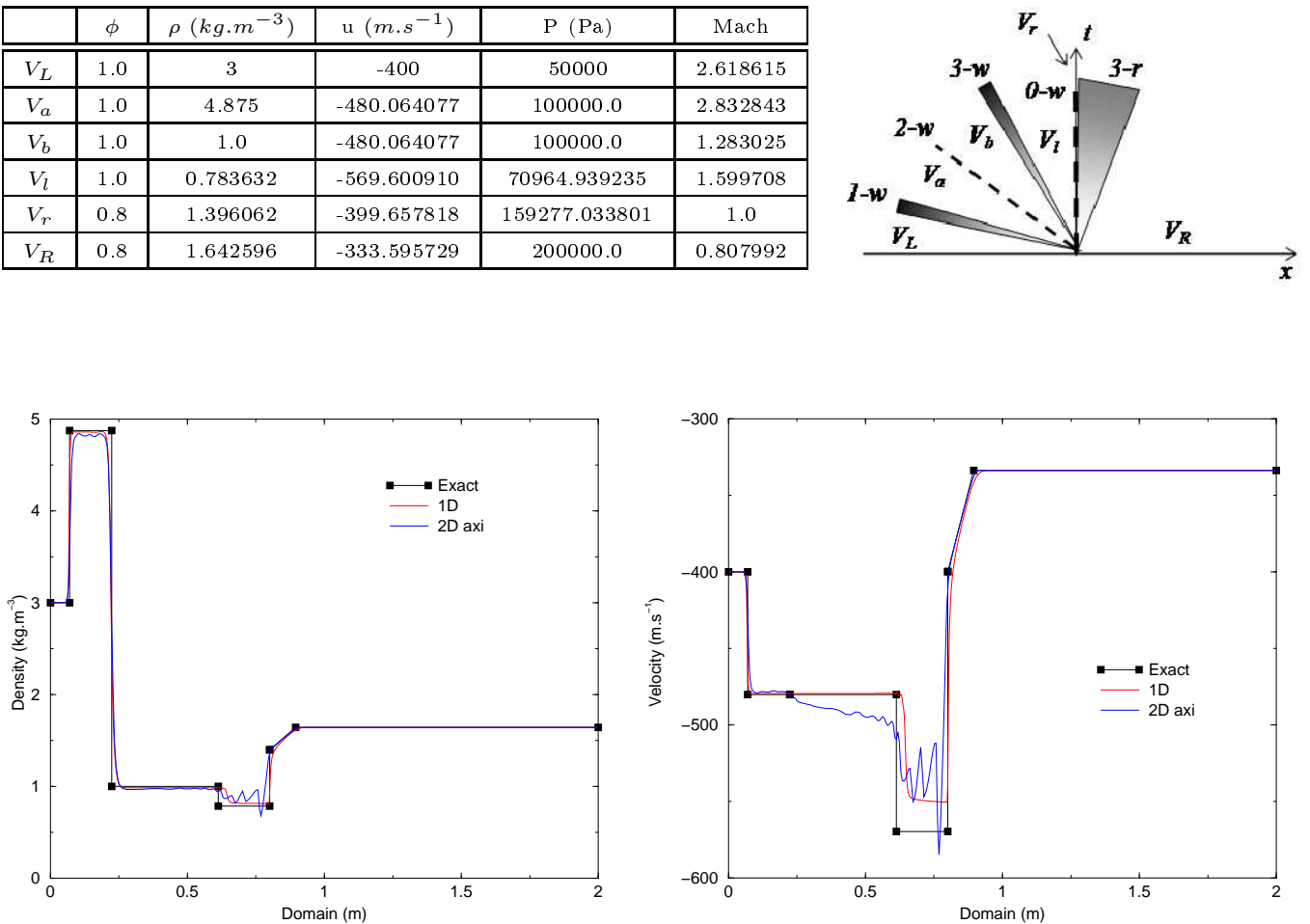

Fig. 29. Configuration of type $R R_{3} \_s c k=\{1-s, 2-w, 3-s, 0-w, 3-r\}$ at time $12.10^{-4} \mathrm{~s}$.

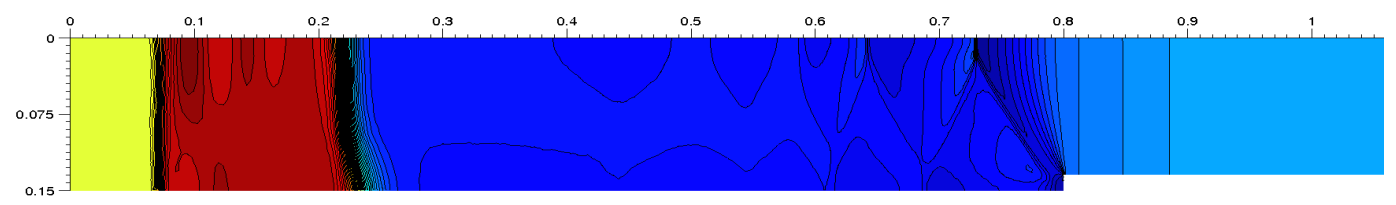

Fig. 30.50 isodensity from 0.4 to 5 at time $12 \cdot 10^{-4} \mathrm{~s}$.

Comments. Table 12 gives the exact solution obtained with the inverse Riemann problem while figures 29 and 30 show respectively the comparison between the exact solution and the numerical approximations for the density and velocity with the two schemes. With this last configuration of the $R R$ group, we observe that the density curve of the 3D-solution fits rather well with the theoretical density curve but the velocity are very poorly approximated. The origin of such a difference seems to be the contact discontinuity which is clearly not preserved by the axisymmetric solution. In this case, it becomes difficult to draw comparisons between the two models although the configurations are really similar. 


\subsection{Resonant wave configurations}

\subsubsection{Configuration $R_{1}$}

Table 13

Configuration of type $R_{1}=\{0-w, 1-s, 0-w, 2-w, 3-r\}$

\begin{tabular}{|c|c|c|c|c|c|}
\hline & $\phi$ & $\rho\left(k g . m^{-3}\right)$ & $\mathrm{u}\left(\mathrm{m.s}^{-1}\right)$ & $\mathrm{P}(\mathrm{Pa})$ & Mach \\
\hline \hline$V_{L}$ & 1.0 & 1 & 500 & 100000 & 1.336306 \\
\hline$V_{s, l}$ & 0.979139 & 1.051791 & 485.507722 & 107325.116343 & 1.284536 \\
\hline$V_{s, r}$ & 0.979139 & 1.565848 & 326.119084 & 188717.365256 & 0.793928 \\
\hline$V_{r}$ & 0.95 & 1.453274 & 362.158786 & 170000.000005 & 0.894920 \\
\hline$V_{a}$ & 0.95 & 1.2 & 362.158786 & 170000.000005 & 0.813207 \\
\hline$V_{R}$ & 0.95 & 1.347710 & 414.461597 & 200000.0 & 0.909292 \\
\hline
\end{tabular}
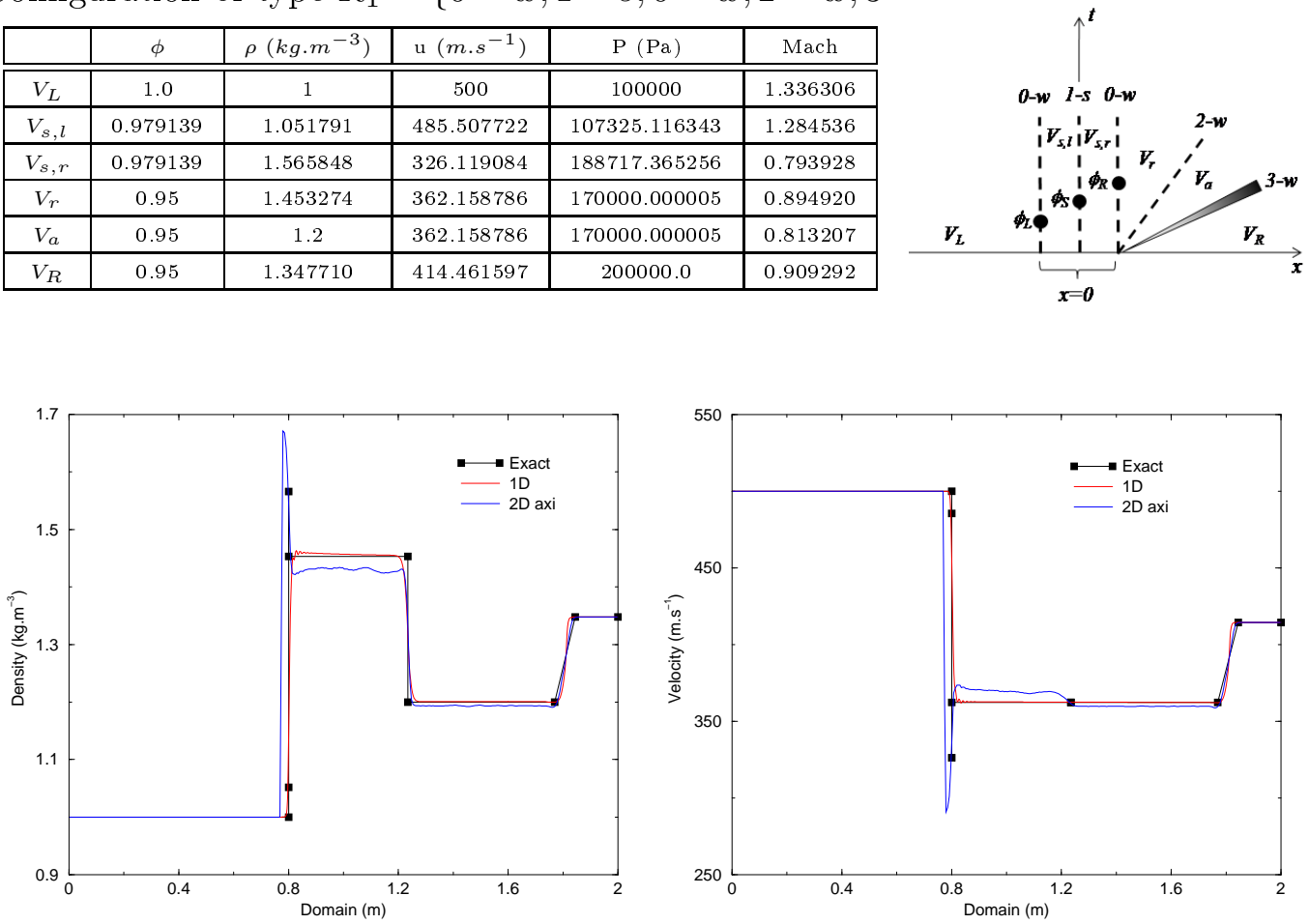

Fig. 31. Configuration of type $R_{1}=\{0-w, 1-s, 0-w, 2-w, 3-r\}$ at time $12 \cdot 10^{-4} s$.

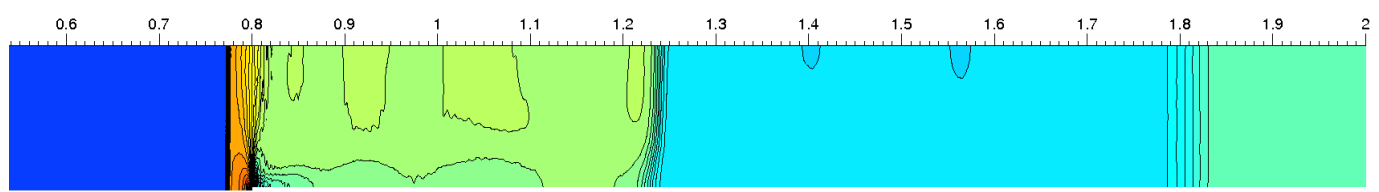

Fig. 32.50 isodensity from 0.8 to 2 at time $12 \cdot 10^{-4} s$.

Comments. Table 13 gives the exact solution obtained with the inverse Riemann problem while figures 31 and 32 show respectively the comparison between the exact solution and the numerical approximations for the density and velocity with the two schemes. In the resonant configuration, the transition between the state $V_{L}$ and $V_{r}$ requires two intermediate states $V_{s, l}$ and $V_{s, r}$ which are superposed with the interface with two different densities (indicated by the black box in figure 31). We indeed observe that the three-dimensional simulation presents a peak at the interface which corresponds to the higher intermediate density. It is remarkable to see that the 1D-exact solution coincides very well with the axisymmetric solution. 


\subsubsection{Configuration $R_{3}$}

Table 14

Configuration of type $R_{3}=\{1-r, 2-w, 0-w, 3-s, 0-w\}$

\begin{tabular}{|c|c|c|c|c|c|}
\hline & $\phi$ & $\rho\left(k g . \mathrm{m}^{-3}\right)$ & $\mathrm{u}\left(\mathrm{m.s}^{-1}\right)$ & $\mathrm{P}(\mathrm{Pa})$ & Mach \\
\hline \hline$V_{L}$ & 0.9 & 1 & -1000 & 400000 & 1.336306 \\
\hline$V_{a}$ & 0.9 & 0.909028 & -929.300937 & 350000 & 1.265747 \\
\hline$V_{l}$ & 0.9 & 0.3 & -929.300937 & 350000 & 0.727142 \\
\hline$V_{s, l}$ & 0.947867 & 0.314418 & -841.908993 & 373773.744392 & 0.652605 \\
\hline$V_{s, r}$ & 0.947867 & 0.148077 & -1787.656974 & 123423.468739 & 1.654876 \\
\hline$V_{R}$ & 1.0 & 0.136386 & -1839.710883 & 110000.000014 & 1.731309 \\
\hline
\end{tabular}
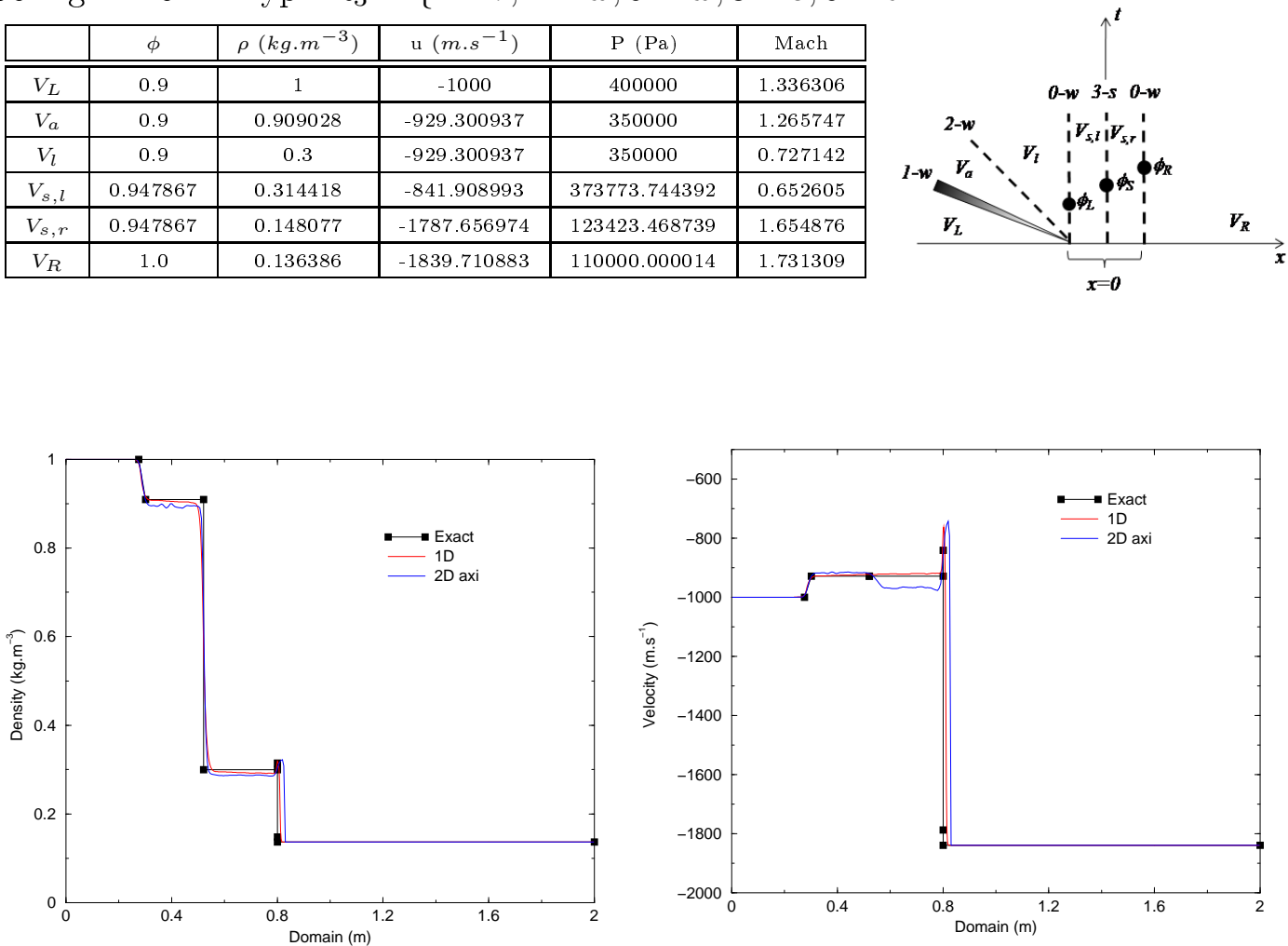

Fig. 33. Configuration of type $R_{3}=\{1-r, 2-w, 0-w, 3-s, 0-w\}$ at time $3.10^{-4} s$.

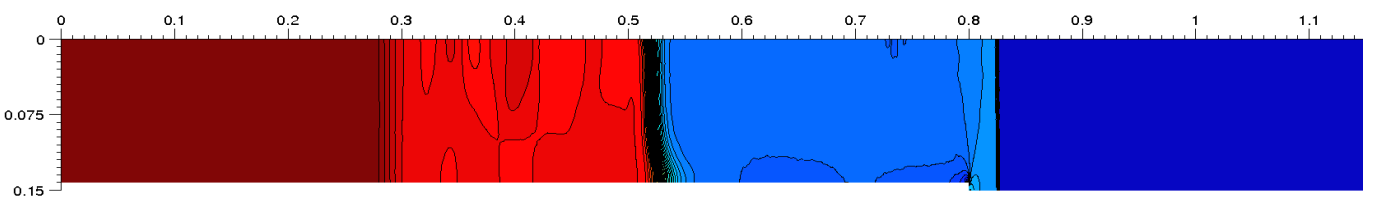

Fig. 34.50 isodensity from 0.1 to 1 at time $3 \cdot 10^{-4} s$.

Comments. Table 14 gives the exact solution obtained with the inverse Riemann problem while figures 33 and 34 show respectively the comparison between the exact solution and the numerical approximations for the density and velocity with the two schemes. We now consider a similar resonant situation but with the $3-s$ stationary shock inside the interface. The three solutions fit nicely and no peak is observed since the intermediate state density $V_{s, l}$, $V_{s, r}$ belongs to the density of $V_{l}$ and $V_{L}$. This point seems to confirm that the peak in the previous $3 \mathrm{D}$ approximation is not a numerical artefact but an approximation of the intermediate state densities. 


\subsection{Resonant and splitting wave configurations}

\subsubsection{Configuration $L R R_{1}$}

Table 15

Configuration of type $L R R_{1}=\{1-r, 0-w, 1-s, 0-w, 2-w, 3-s\}$

\begin{tabular}{|c|c|c|c|c|c|}
\hline & $\phi$ & $\rho$ & $\mathrm{u}$ & $\mathrm{P}$ & Mach \\
\hline \hline$V_{L}$ & 1.3 & 1.862000 & 0.826000 & 2.458300 & 0.607559 \\
\hline$V_{l}$ & 1.3 & 1.327678 & 1.270616 & 1.531063 & 1.0 \\
\hline$V_{s, l}$ & 1.31102 & 1.214478 & 1.377370 & 1.351478 & 1.103511 \\
\hline$V_{s, r}$ & 1.31102 & 1.427126 & 1.172136 & 1.694791 & 0.909049 \\
\hline$V_{r}$ & 1.6 & 1.789307 & 0.766029 & 2.326095 & 0.567819 \\
\hline$V_{a}$ & 1.6 & 2.0 & 0.766029 & 2.326095 & 0.600320 \\
\hline$V_{R}$ & 1.6 & 1.795636 & 0.629806 & 2.0 & 0.504356 \\
\hline
\end{tabular}
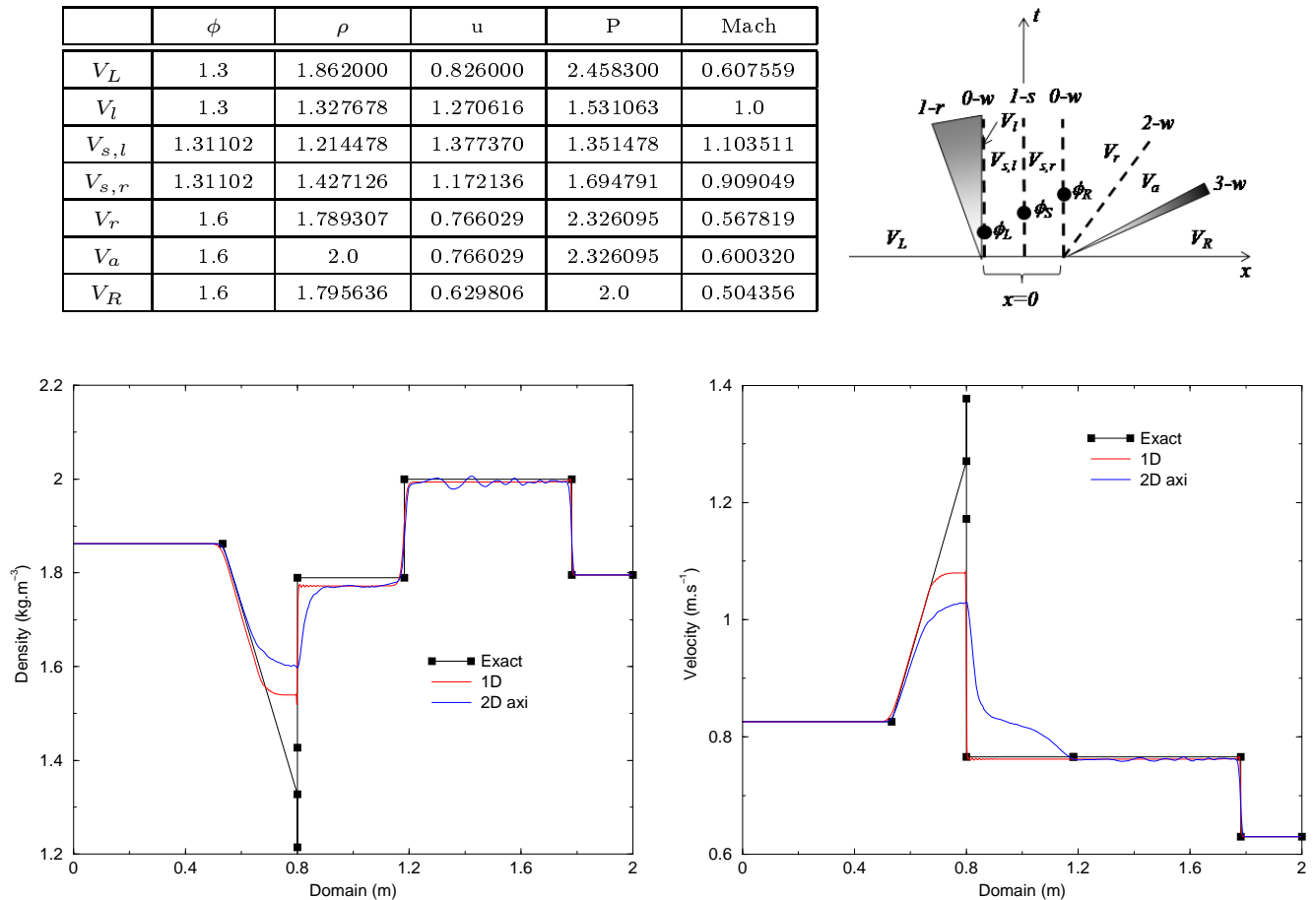

Fig. 35. Configuration of type $L R R_{1}=\{1-r, 0-w, 1-s, 0-w, 2-w, 3-s\}$ at time $0.5 s$.

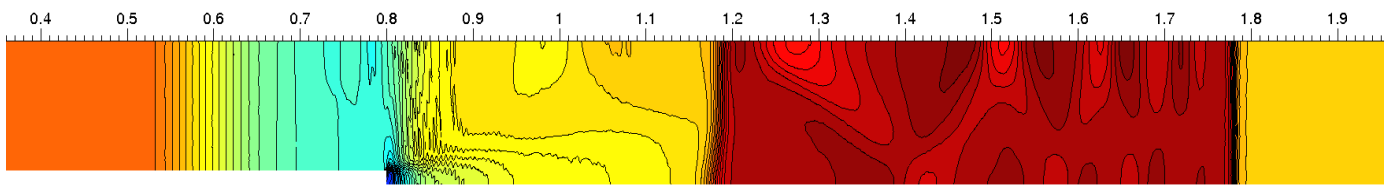

Fig. 36. 50 isodensity from 1.3 to 2 at time $0.5 s$.

Comments. Table 15 gives the exact solution obtained with the inverse Riemann problem while figures 35 and 36 show respectively the comparison between the exact solution and the numerical approximations for the density and velocity with the two schemes. We here deal with the more complex situation where we have both a splitting wave and a resonant situation. The positive point is that we obtain the same theoretical configuration both with the one-dimensional and the three-dimensional model which suggest that such a configuration is physical. The negative point is the very poor approximation of the $1-r$ rarefaction on the left side of the interface that reaches the sonic point. We have performed numerical simulations with finer meshes with the axisymmetric model to see if, possibly, we better catch the rarefaction but the tests were negative. 


\subsubsection{Configuration $\mathrm{LRR}_{3}$}

Table 16

Configuration of type $L R R_{3}=\{1-s, 2-w, 3-r, 0-w, 3-s, 0-w\}$

\begin{tabular}{|c|c|c|c|c|c|}
\hline & $\phi$ & $\rho\left(k g . \mathrm{m}^{-3}\right)$ & $\mathrm{u}\left(\mathrm{m} . \mathrm{s}^{-1}\right)$ & $\mathrm{P}(\mathrm{Pa})$ & Mach \\
\hline \hline$V_{L}$ & 0.95 & 1.6 & -200.0 & 100000 & 0.676123 \\
\hline$V_{a}$ & 0.95 & 2.6 & -355.043418 & 200000 & 1.081905 \\
\hline$V_{b}$ & 0.95 & 3.0 & -355.043418 & 200000 & 1.162152 \\
\hline$V_{l}$ & 0.95 & 3.427892 & -313.761442 & 241045.084752 & 1.0 \\
\hline$V_{s, l}$ & 0.968138 & 2.987938 & -353.216986 & 198875.088944 & 1.157106 \\
\hline$V_{s, r}$ & 0.968138 & 3.786653 & -278.713217 & 277505.646851 & 0.870133 \\
\hline$V_{R}$ & 1.0 & 4.052357 & -252.140550 & 305143.954267 & 0.776569 \\
\hline
\end{tabular}
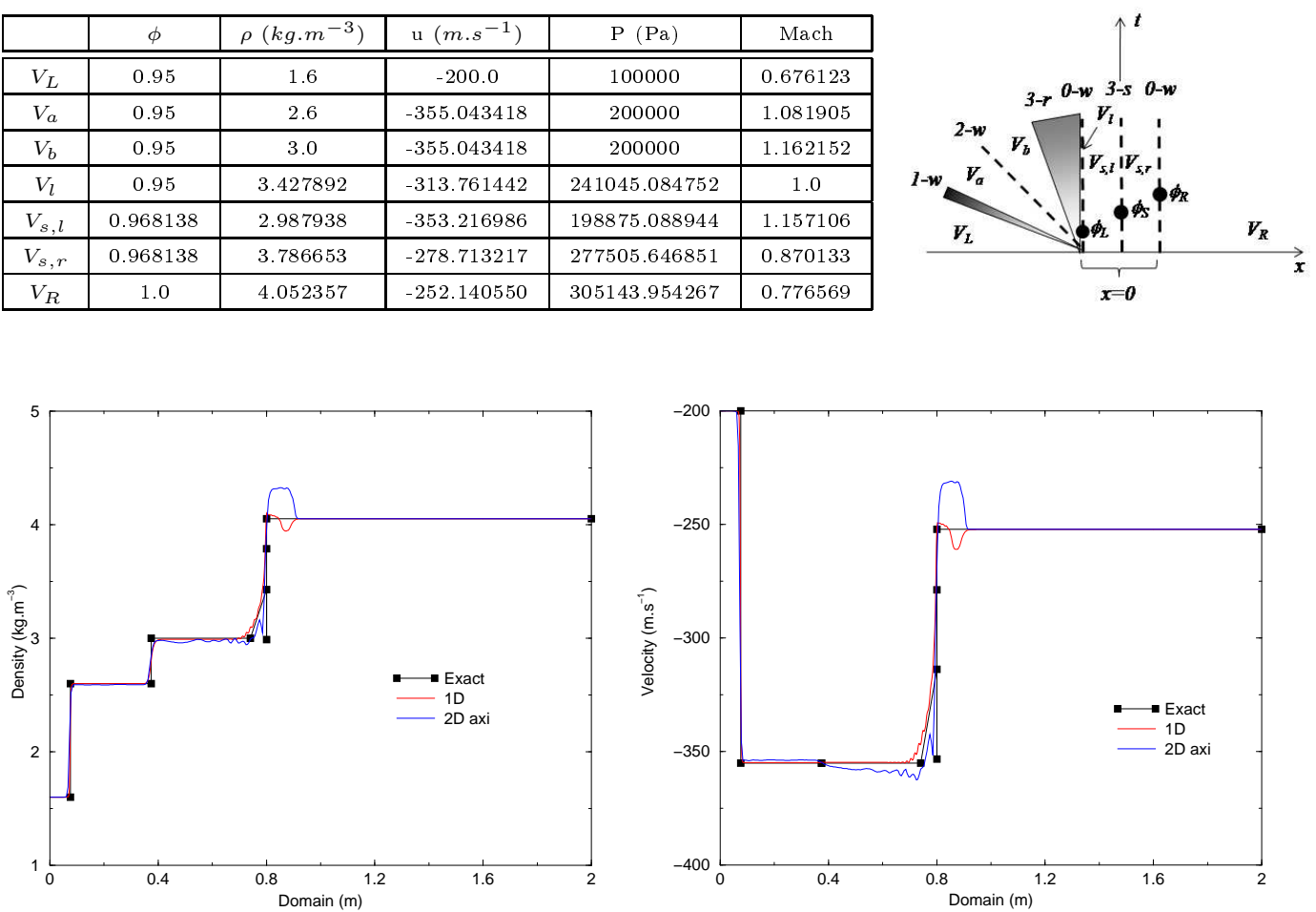

Fig. 37. Configuration of type $L R R_{3}=\{1-s, 2-w, 3-r, 0-w, 3-s, 0-w\}$ at time $12 \cdot 10^{-4} \mathrm{~s}$.

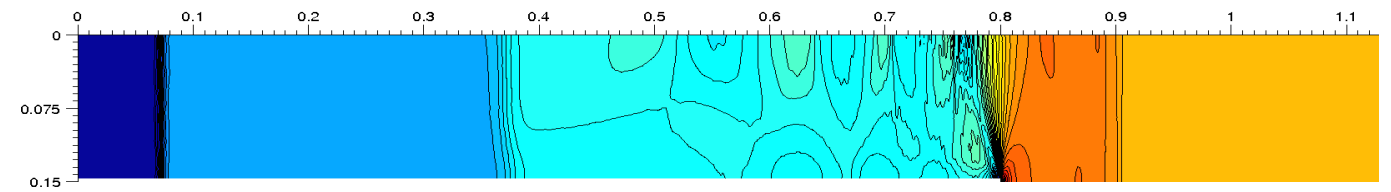

Fig. 38. 50 isodensity from 1.6 to 5.2 at time $12.10^{-4} \mathrm{~s}$.

Comments. Table 16 gives the exact solution obtained with the inverse Riemann problem while figures 37 and 38 show respectively the comparison between the exact solution and the numerical approximations for the density and velocity with the two schemes. This test was performed with a small variation of $\phi$ so the $3-r$ rarefaction from $V_{b}$ to the sonic state $V_{s, l}$ is small, nevertheless, we observe an overshoot for the axisymmetric simulation and oscillations with the $1 \mathrm{D}$-solution which indicates that such a configuration is really difficult to compute. The positive point is that the three solutions correspond to the same configuration and state $V_{a}$ is well-approached. Note that the density of the intermediate state $V_{s, l}$ is lower than $V_{l}$ and $V_{b}$. We observe that both the $1 \mathrm{D}$ and $3 \mathrm{D}$ approximations try to catch the lower density, we think that such a behaviour of the scheme is responsible of a wrong evaluation of the rarefaction up to the sonic state. 


\subsubsection{Configuration $R R R_{1}$}

Table 17

Configuration of type $R R R_{1}=\{0-w, 1-s, 0-w, 1-r, 2-w, 3-r\}$

\begin{tabular}{|c|c|c|c|c|c|}
\hline & $\phi$ & $\rho$ & $\mathrm{u}$ & $\mathrm{P}$ & Mach \\
\hline \hline$V_{L}$ & 1.0 & 1.3 & 2 & 1 & 1.927248 \\
\hline$V_{s, l}$ & 0.78177 & 1.872903 & 1.775738 & 1.667250 & 1.590641 \\
\hline$V_{s, r}$ & 0.78177 & 3.775791 & 0.880818 & 4.643562 & 0.671275 \\
\hline$V_{r}$ & 0.7 & 2.969906 & 1.250641 & 3.318027 & 1.0 \\
\hline$V_{a}$ & 0.7 & 0.533582 & 3.067818 & 0.3 & 3.457843 \\
\hline$V_{b}$ & 0.7 & 1.0 & 3.067818 & 0.3 & 4.733745 \\
\hline$V_{R}$ & 0.7 & 2.363115 & 3.675948 & 1.0 & 4.775818 \\
\hline
\end{tabular}
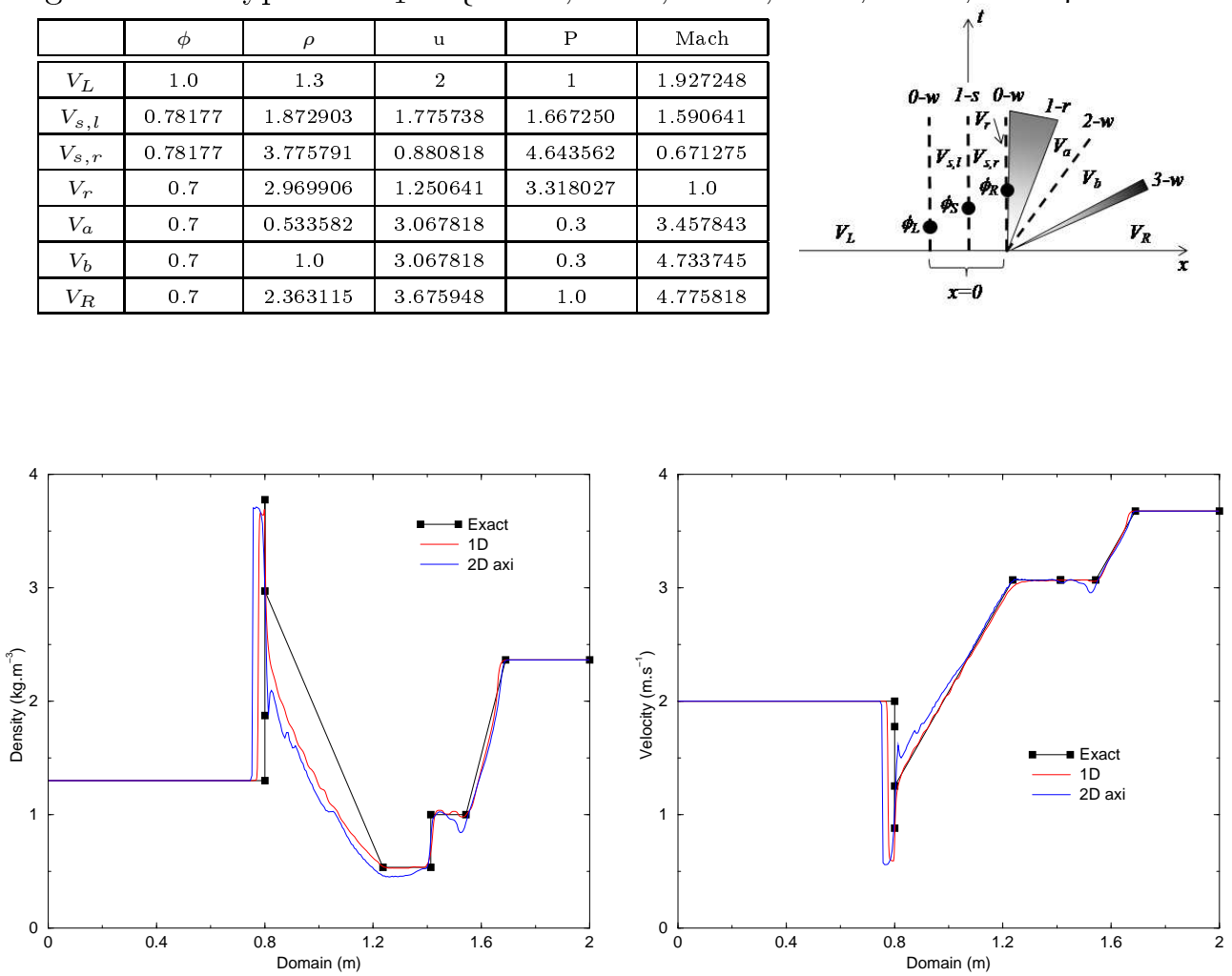

Fig. 39. Configuration of type $R R R_{1}=\{0-w, 1-s, 0-w, 1-r, 2-w, 3-r\}$ at time $0.2 \mathrm{~s}$.

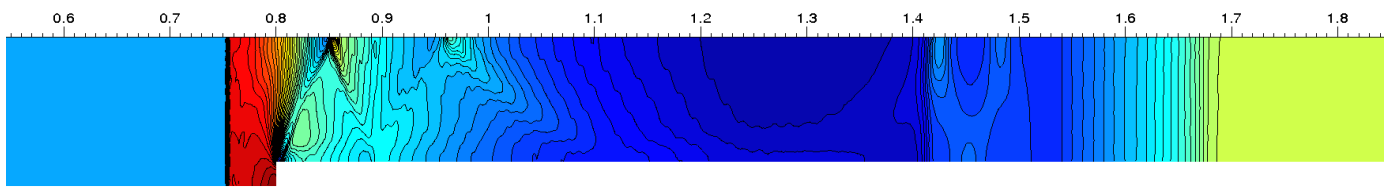

Fig. 40.50 isodensity from 0.3 to 4 at time $0.2 s$.

Comments. Table 17 gives the exact solution obtained with the inverse Riemann problem while figures 39 and 40 show respectively the comparison between the exact solution and the numerical approximations for the density and velocity with the two schemes. We first mention that we have drawn a simple line between $V_{r}$ and $V_{a}$ for the sake of simplicity to represent the rarefaction and the real curve would be a convex one very similar to the numerical approximations. For the present case, the intermediate value $V_{s, r}$ density is higher than the other density and we find again that both the numerical approximations try to catch the higher density value. 


\subsubsection{Configuration $R_{R} R_{3}$}

Table 18

Configuration of type $R R R_{3}=\{1-s, 2-w, 0-w, 3-s, 0-w, 3-r\}$

\begin{tabular}{|c|c|c|c|c|c|}
\hline & $\phi$ & $\rho$ & $\mathrm{u}$ & $\mathrm{P}$ & Mach \\
\hline \hline$V_{L}$ & 1.0 & 1.4 & -2.0 & 2.0 & 1.414214 \\
\hline$V_{a}$ & 1.0 & 2.077419 & -2.591083 & 3.5 & 1.687118 \\
\hline$V_{l}$ & 1.0 & 0.3 & -2.591083 & 3.5 & 0.641127 \\
\hline$V_{s, l}$ & 0.889412 & 0.258647 & -3.379023 & 2.843740 & 0.861264 \\
\hline$V_{s, r}$ & 0.889412 & 0.200487 & -4.359267 & 1.987030 & 1.170281 \\
\hline$V_{r}$ & 0.87 & 0.232799 & -3.837976 & 2.449388 & 1.0 \\
\hline$V_{R}$ & 0.87 & 0.269081 & -3.273959 & 3.0 & 0.828687 \\
\hline
\end{tabular}
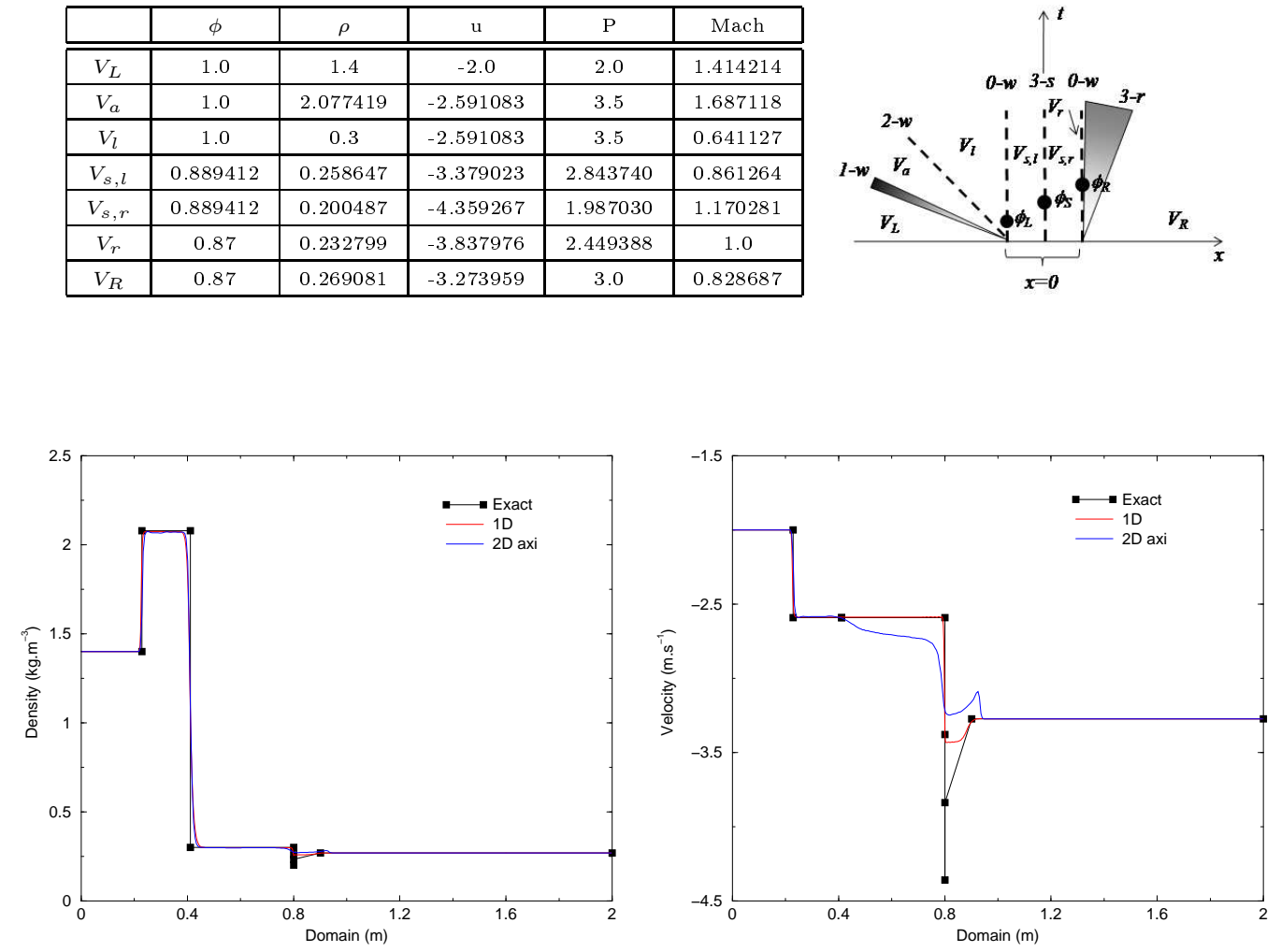

Fig. 41. Configuration of type $R R R_{3}=\{1-s, 2-w, 0-w, 3-s, 0-w, 3-r\}$ at time $0.15 s$.

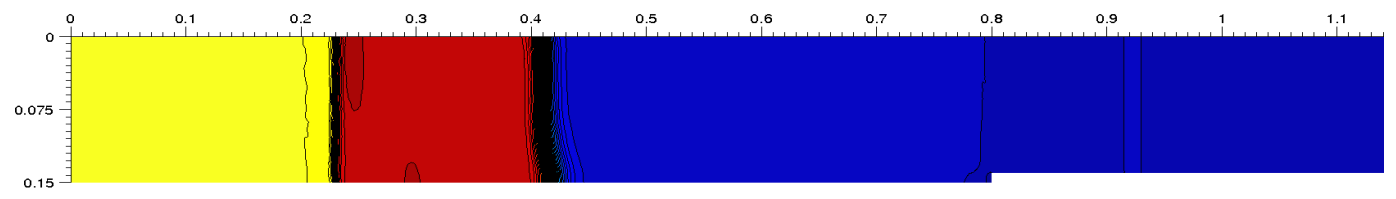

Fig. 42.50 isodensity from 0.2 to 2.2 at time $0.15 \mathrm{~s}$.

Comments. Table 18 gives the exact solution obtained with the inverse Riemann problem while figures 41 and 42 show respectively the comparison between the exact solution and the numerical approximations for the density and velocity with the two schemes. The configuration $R R R_{3}$ is similar to the $L R R_{1}$ one (in particular the velocity). The $3-r$ transition to the $3 \mathrm{D}$ sonic state at the interface is poorly approximated where this time the schemes attempt to catch the higher velocity between the three states $V_{s, l}, V_{s, r}$ and $V_{r}$. Density variations are too small to comment the simulations around the interface but the other states $V_{a}, V_{l}$ are well-approximated. 


\section{Non uniqueness of the Riemann problem solutions}

Non uniqueness of the Riemann problem solutions is well-known for the nonconservative Euler system [3] and the nonconservative shallow-water problem [2]. The point is that the transition across the interface does not behave in the same manner whether we deal with a subsonic or a supersonic flow. Consequently, we can exhibit Riemann problems with two distinct entropy solutions (in the Lax sense) whether we use the subsonic branch of the interface transition or the supersonic one. We propose here several couples of configurations which are solutions of the same Riemann problem and we compare the 1Dsolution with the numerical approximation obtained with the axisymmetric model.

\subsection{Non uniqueness between configurations $C$ and $D$}

We first test the configuration proposed by Andrianov and Warnecke in [3]. We have listed the densities, velocities and pressures obtained by the inverse Riemann problem in table 19.

Table 19

Configuration of type $C$ and $D$

\begin{tabular}{|c|c|c|c|c|}
\hline & $\phi$ & $\rho$ & $\mathrm{u}$ & $\mathrm{P}$ \\
\hline \hline \multicolumn{5}{|c|}{ Configuration of type $C=\{1-s, 0-w, 2-w, 3-s\}$} \\
\hline$V_{L}$ & 0.8 & 0.2069 & 3.991 & 0.07 \\
\hline$V_{l}$ & 0.8 & 1.1109 & 0.3377 & 3.4634 \\
\hline$V_{r}$ & 0.3 & 1.0019 & 0.9985 & 2.9972 \\
\hline$V_{a}$ & 0.3 & 0.6997 & 0.9985 & 2.9972 \\
\hline$V_{R}$ & 0.3 & 0.1354 & -3.1668 & 0.0833 \\
\hline Configuration of type $D=\{0-w, 1-s, 2-w, 3-s\}$ \\
\hline$V_{L}$ & 0.8 & 0.2069 & 3.991 & 0.07 \\
\hline$V_{r}$ & 0.3 & 0.5736 & 3.8387 & 0.2918 \\
\hline$V_{a}$ & 0.3 & 2.4112 & 1.6389 & 3.934 \\
\hline$V_{b}$ & 0.3 & 0.724 & 1.6389 & 3.934 \\
\hline$V_{R}$ & 0.3 & 0.1354 & -3.1668 & 0.0833 \\
\hline
\end{tabular}

With the same initial conditions, the Riemann problem for the nonconservative model has two distinct entropy solutions in the Lax sense but the 
numerical scheme based on the nonconservative Rusanov flux [11] and the numerical scheme based on the axisymmetric model provide the same configuration $C$. Figures 43 give the theoretical density and the entropy using the inverse Riemann problem and the numerical approximations obtained with the one-dimensional model and the axisymmetric model (at $r=0$ ). We present in figure 44 the repartition of the density and entropy at the same time for the full three-dimensional model. We observe that the shock are longitudinal which guarantees a good correspondence between the one-dimensional nonconservative model and the three-dimensional conservative one.
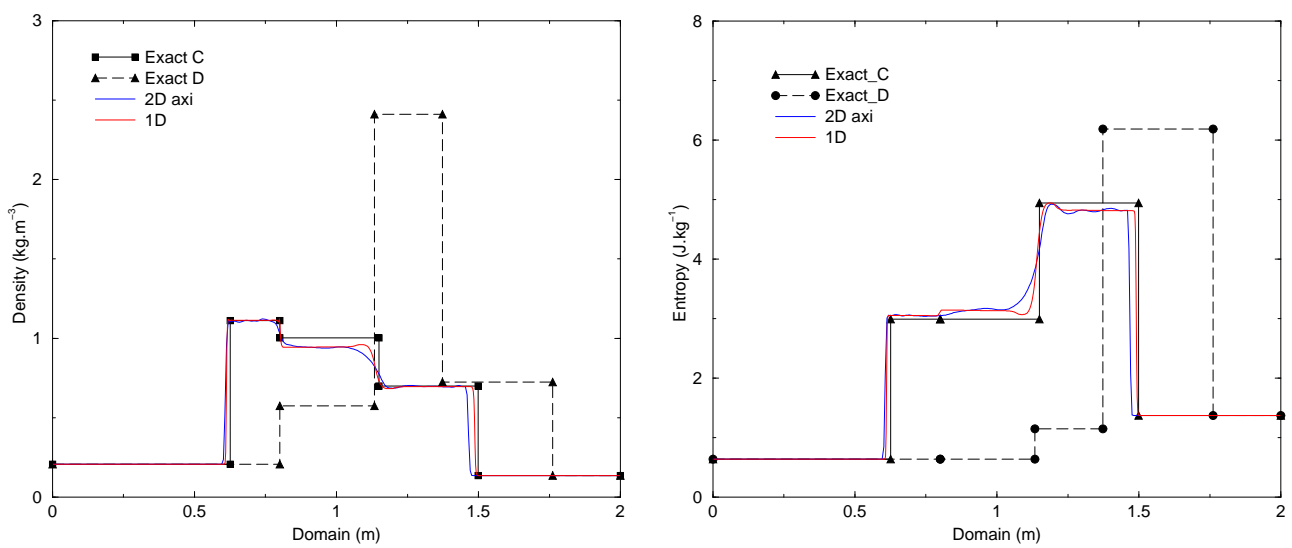

Fig. 43. Distribution of the density and the entropy obtained by the nonconservative and the axisymmetric models and the two solutions of the inverse Riemann problem at time $t=0.35 \mathrm{~s}$.
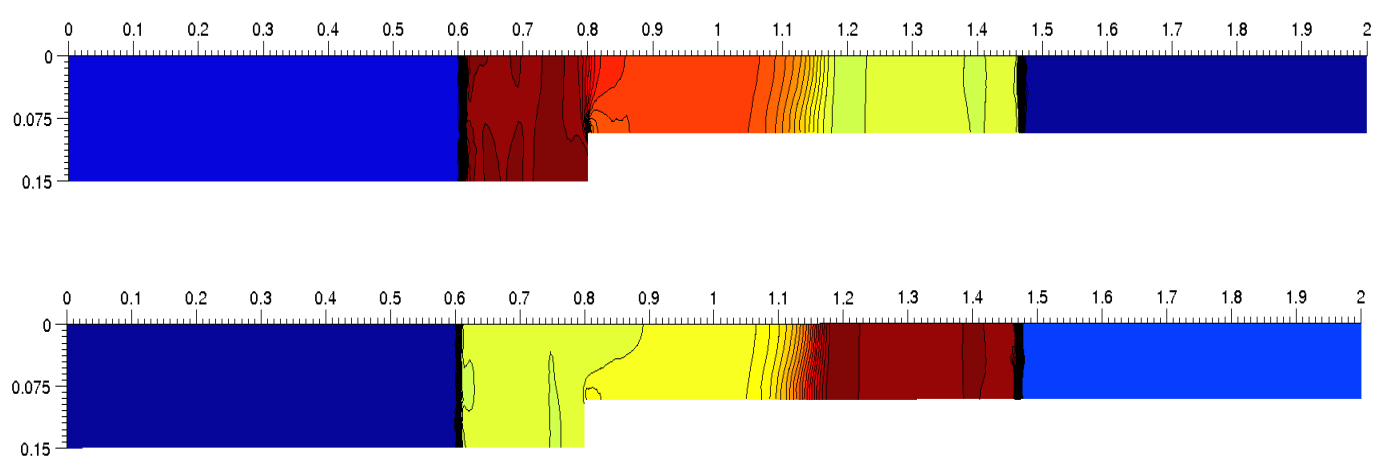

Fig. 44. 50 isodensity (top) from 0.135 to 1.13 and 50 isoentropy (bottom) from 0.635 to 4.92 at time $t=0.35 \mathrm{~s}$.

We plot the velocity and the Mach number in figure 45 for the theoretical solutions (configuration $C$ and $D$ ), the one-dimensional approximation and the axisymmetric model solution at $r=0$ while we give in figures 46 the map of the velocity and the Mach number using the axisymmetric model. Like the density map, the velocity obtained by the axisymmetric model presents very straight longitudinal shocks which legitimate the one-dimensional approach. 

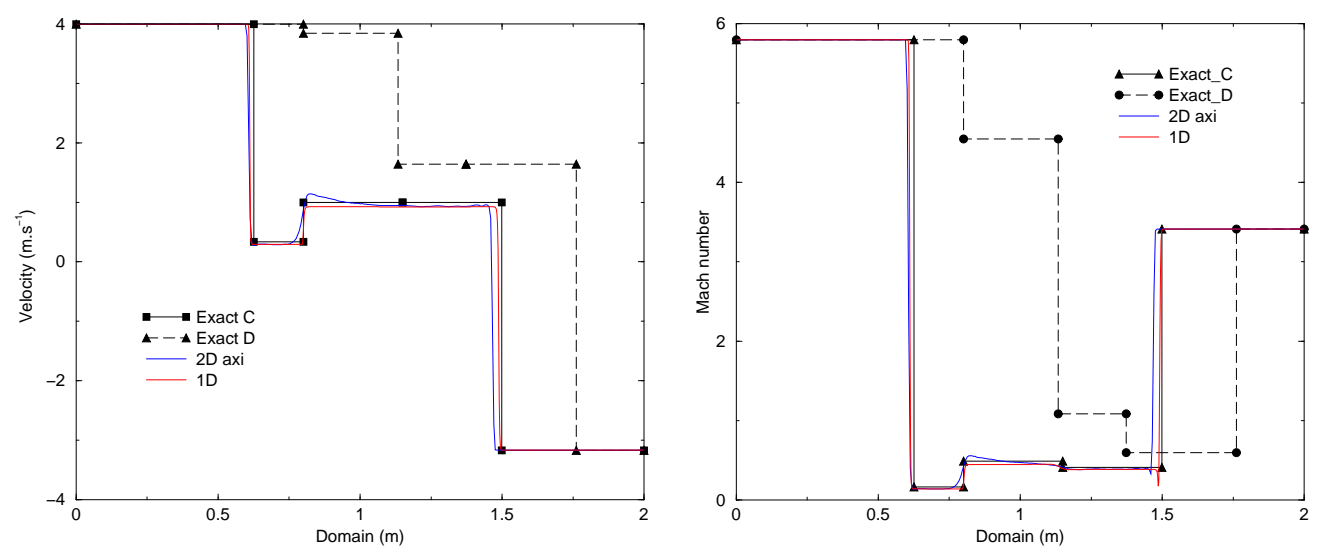

Fig. 45. Distribution of the velocity and the Mach number obtained by the nonconservative and the axisymmetric models and the two solutions of the inverse Riemann problem at time $t=0.35 \mathrm{~s}$.
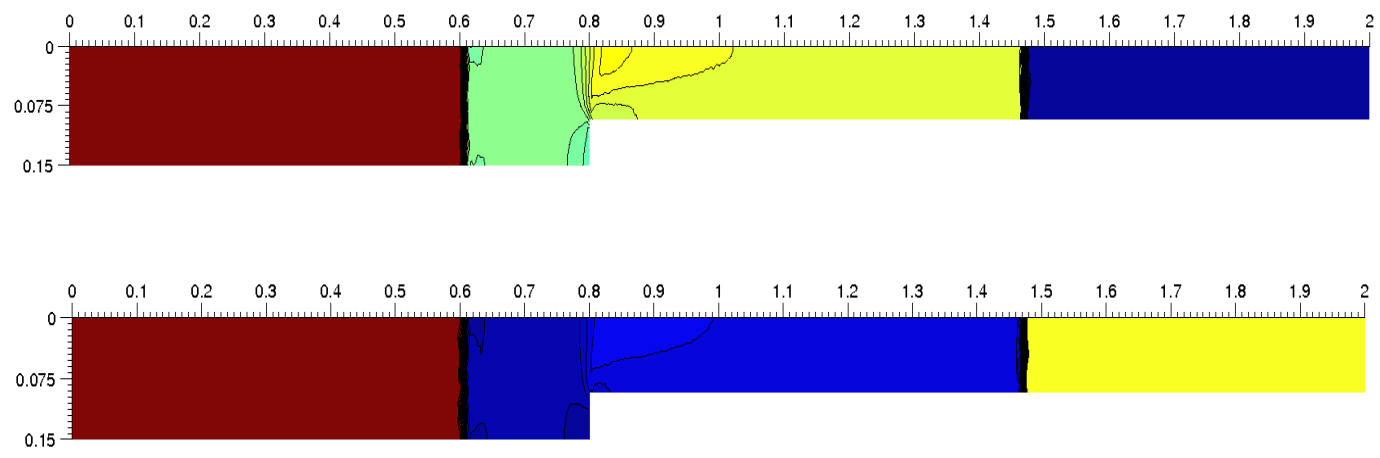

Fig. 46. 50 isovelocity (top) from -3.17 to 3.99 and 50 isomach (bottom) from 0.0179 to 5.8 at time $t=0.35 \mathrm{~s}$.

\subsection{Non uniqueness between configurations $D$ and $R R R 1$}

We proposed here a new non uniqueness case between configurations $D$ and $R R R_{1}$. Densities, velocities and pressures for the two configurations obtained by the inverse Riemann problem are presented in table 20 .

With the same initial conditions, the Riemann problem for the nonconservative model has two distinct entropy solutions in the Lax sense but the numerical scheme based on the nonconservative Rusanov flux [11] selects the $D$ configuration whereas the numerical solution based on the axisymmetric model [12] corresponds to the $R R R_{1}$ configuration. We display in figure 47 the theoretical density and entropy using the inverse Riemann problem and the two numerical solutions (with the one-dimensional and the axisymmetric models). We note that the physical solution obtained with the 3D-model corresponds 
Table 20

Configuration of type $\mathrm{D}$ and $R R R_{1}$

\begin{tabular}{|c|c|c|c|c|}
\hline & $\phi$ & $\rho\left(k g . m^{-3}\right)$ & $\mathrm{u}\left(m_{.} s^{-1}\right)$ & $\mathrm{P}(\mathrm{Pa})$ \\
\hline \hline \multicolumn{5}{|c|}{ Configuration of type $D=\{0-w, 1-s, 2-w, 3-s\}$} \\
\hline$V_{L}$ & 1.0 & 1 & 650 & 60000 \\
\hline$V_{r}$ & 0.75 & 1.4557 & 595.3377 & 101502.1874 \\
\hline$V_{a}$ & 0.75 & 0.7469 & 790.5361 & 39874.3 \\
\hline$V_{b}$ & 0.75 & 4.9888 & 790.5361 & 39874.3 \\
\hline$V_{R}$ & 0.75 & 9.2747 & 860.373 & 95000 \\
\hline Configuration of type $R R R_{1}=\{0-w, 1-s, 0-w, 1-r, 2-w, 3-r\}$ \\
\hline$V_{L}$ & 1.0 & 1 & 650 & 60000 \\
\hline$V_{s, l}$ & 0.940495 & 1.0802 & 639.7901 & 66846.4501 \\
\hline$V_{s, r}$ & 0.940495 & 3.1490 & 219.4730 & 357338.2303 \\
\hline$V_{r}$ & 0.75 & 2.3128 & 374.7221 & 231970.8357 \\
\hline$V_{a}$ & 0.75 & 0.6589 & 790.7742 & 40000 \\
\hline$V_{b}$ & 0.75 & 5 & 790.7742 & 40000 \\
\hline$V_{R}$ & 0.75 & 9.2747 & 860.373 & 95000 \\
\hline
\end{tabular}

to the largest global entropy production (global in the sense that we spatially integrate the entropy on interval $[0,2])$. Such a criterion has been mentioned by Andrianov and Warnecke [3].
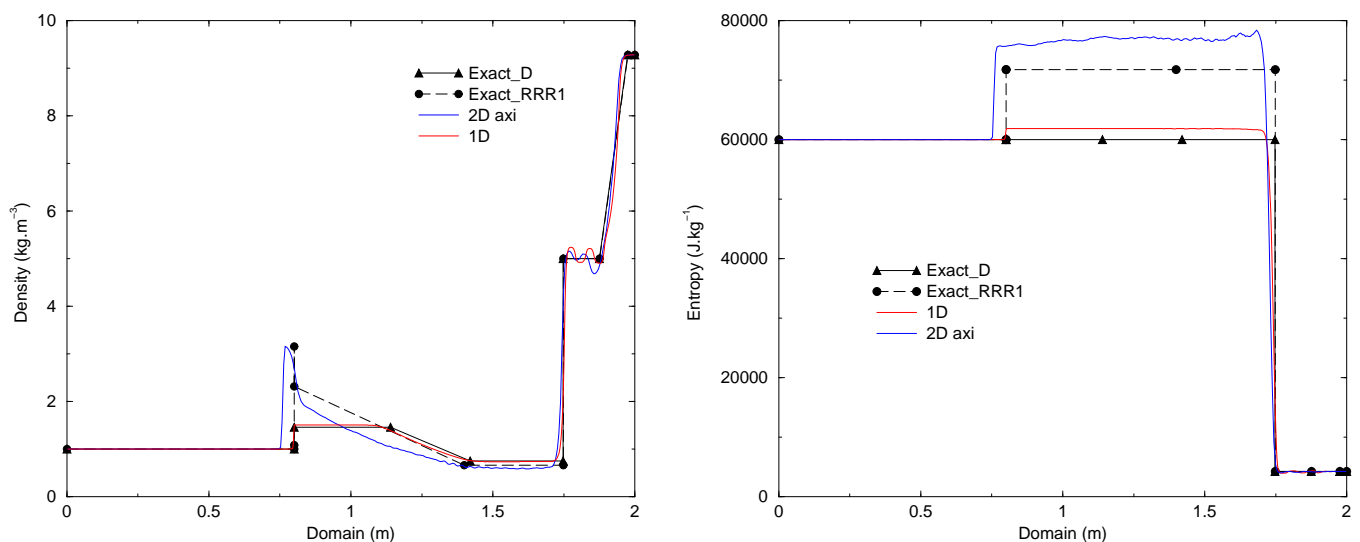

Fig. 47. Distribution of the density and the entropy obtained by the nonconservative and the axisymmetric models and the two solutions of the inverse Riemann problem at time $t=1 \mathrm{~ms}$.

We show in figure 48 the density and the entropy maps obtained with the 
axisymmetric model. We observe that the shocks are mainly longitudinal which explains the nice fitting between the numerical solution and configuration $R R R_{1}$.
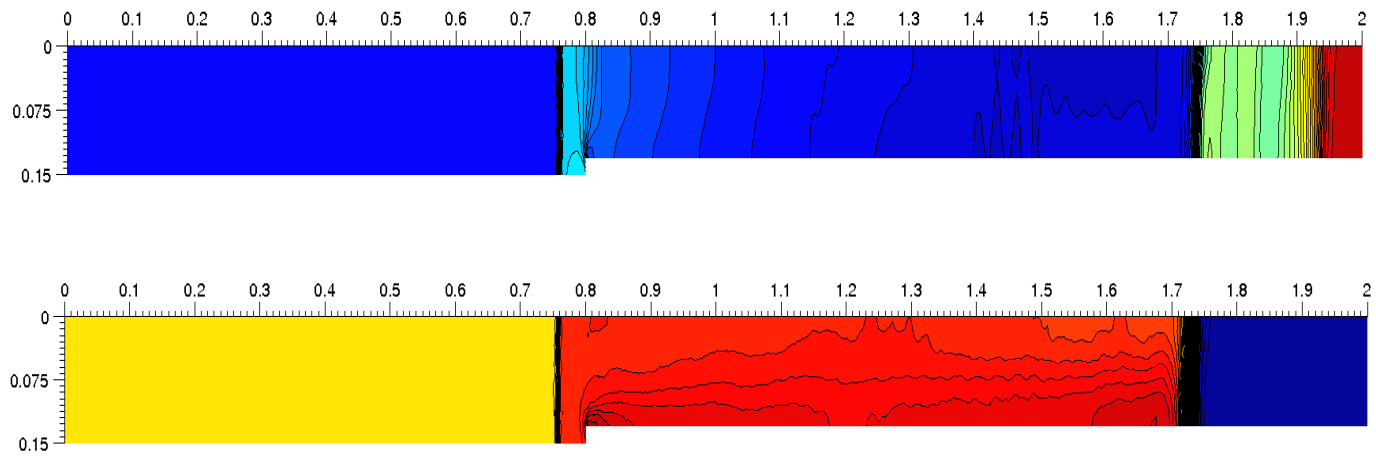

Fig. 48. 50 isodensity (top) from 0 to 10 and 50 isoentropy (bottom) from 0 to 80000 at time $t=1 \mathrm{~ms}$.

We plot in figure 49 the velocity and the Mach number for the theoretical solutions for configurations $D$ and $R R R_{1}$ and the numerical approximation using the $1 \mathrm{D}$-nonconservative model and the $3 \mathrm{D}$-conservative one. We observe a very good correspondence between the $1 \mathrm{D}$-model and configuration $D$ whereas the approximations obtained with the axisymmetric model suit well with configuration $R R R_{1}$.
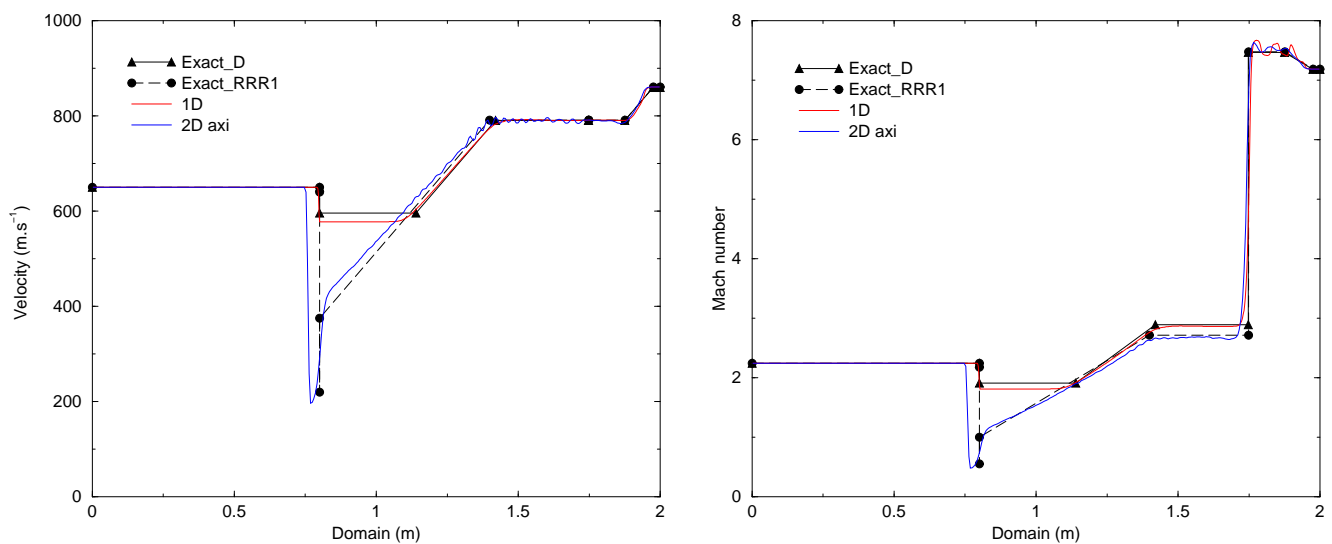

Fig. 49. Distribution of the velocity and the Mach number obtained by the nonconservative and the axisymmetric models and the two solutions of the inverse Riemann problem at time $t=1 \mathrm{~ms}$.

Figure 50 represents the velocity and Mach number maps at time $t=1 \mathrm{~ms}$. Like the density and the entropy, shock waves are longitudinal which guarantee a good correspondence with the theoretical one-dimensional model. 

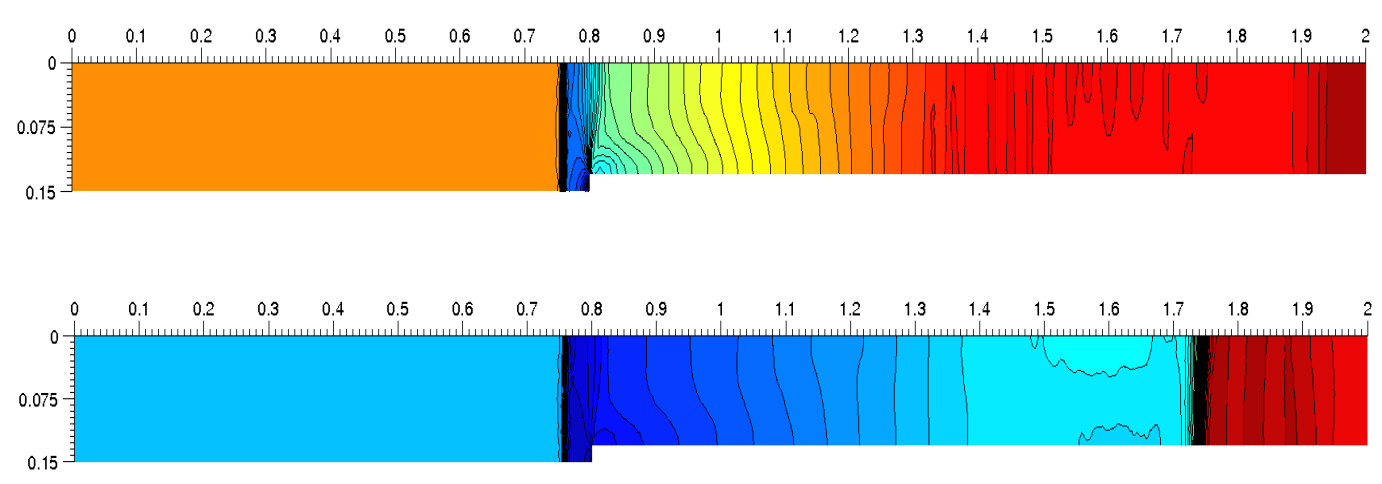

Fig. 50. 50 isovelocity (top) from 0 to 900 and 50 isomach (bottom) from 0 to 8 at time $t=1 \mathrm{~ms}$.

\subsection{Remarks and conjunctures}

We propose here some remarks and conjunctures based on our numerical experience. We do not have clear theoretical arguments or solid experimental facts to prove the conjunctures but just some intuitions upon the complex problem of non uniqueness.

- Since we do not have uniqueness of the solutions with configurations $D$ and $C$ but also with configurations $D$ and $R R R_{1}$, we should have non uniqueness situations with configurations $A$ and $B$ but also with configurations $A$ and $\mathrm{LRR}_{3}$.

- We also think that they may have many other situations of non uniqueness, for example between configurations $D$ and $R R_{1}$ (very similar to $D$ and $R R R_{1}$ ) but also $D$ and $L R_{1}$. Non uniqueness with three available configurations like $D, R R R_{1}$ and $R R_{1}$ seems also conceivable.

- When the solutions of the nonconservative problem are not unique, it has been noticed that two different numerical methods can provide two different solutions for the one-dimensional problem (see [2] for the shallow water problem). A first conclusion could be that scheme $S 1$ chooses the right solution while scheme $S 2$ chooses the wrong one. We think that the numerical schemes do not behave like that and we propose here an other explanation. Assume that for very large velocity $u_{L}$ on the left side, we have a unique supersonic solution corresponding to configuration $D$. If we reduce the velocity $u_{L}$ till a limit velocity $u_{l i m}$ the non uniqueness situation arises and two configurations are now available $D$ and $R R R 1$ for $u_{L}<u_{l i m}$. The axisymmetric model simulation provides the $R R R_{1}$ solution but the numerical simulation with a scheme $S 1$ provides configuration $D$. We think that scheme $S 1$ will provide a $D$ configuration as long as $u_{L}$ is larger to a critical velocity $u_{c}$ : for $u_{L}>u_{c}$ the scheme $S 1$ provides a $D$ configuration and for $u_{L}<u_{c}$ it 
switches to the "good" configuration $R R R_{1}$.

The value of $u_{c}$ depends on the scheme $S 1$ and if we consider an other scheme $S 2$ for the one-dimensional case, the critical velocity where it switches from the wrong configuration to the right configuration may be different. Our view is the following: some schemes have critical velocity close to the limit velocity $u_{c} \approx u_{\text {lim }}$, hence they rapidly behave as the axisymmetric model while other schemes have a critical velocity far from the limit velocity $u_{c}<<u_{l i m}$ and they provide the wrong solution for a larger number of initial conditions.

As a conclusion, we think that a numerical flux does not always provide the right or the wrong solution but provides the right or the wrong solutions for two distinct sets of initial conditions in the phase space. 


\section{Shock wave interaction with a cross-section reduction in a duct}

In numerous engineering applications [5,26], practical situations do not exactly correspond to pure Riemann problems for the nonconservative Euler system with two definitively different states on the left and right side of a crosssection discontinuity. Indeed, in many applications, the state discontinuity and the cross-section jump are not located at the same place (see figure 51). A Riemann problem for the conservative Euler system generates travelling waves (an explosion generated by a high pressure and a low pressure chambers separated by a diaphragm for instance) which interact with the cross-section jump located after the diaphragm. The incident shock wave is then separated into a transmitted shock wave and a reflected shock wave in function of the section variation.
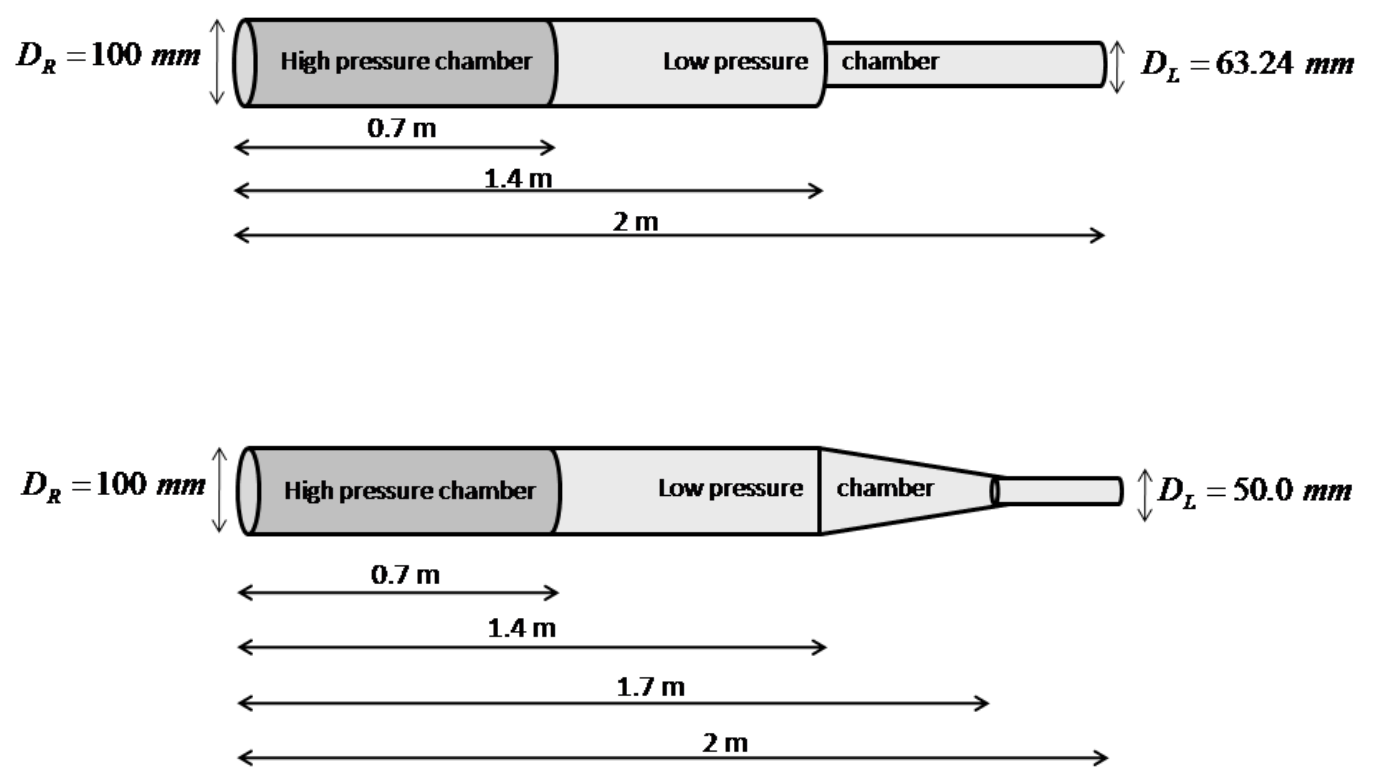

Fig. 51. Shock tube geometry for the test 1 (top) and test 2 (bottom).

In order to perform numerical simulations of such a situation, we consider a shock tube of length $x=[0: 2]$ equipped with a section reduction situated at $x=1.4 \mathrm{~m}$. The initial discontinuity between the high pressure chamber and the low pressure chamber is located at $x=0.7 \mathrm{~m}$. As in the previous sections, we compare the waves evolution between the one-dimensional nonconservative model and the three-dimensional axisymmetric model. We have carried out two kinds of simulation whether we use a discontinuous or a smooth transition between the two sections as displayed in figure 51. We sum up here the two configurations we deal with. Note that the classical Riemann problem we use to generate the shock wave is exactly the same in the two situations in order 
to compare the transition effects.

- A discontinuous cross-section reduction (test 1), i.e. an abrupt transition from $\phi=1$ to $\phi=0.4$ (respectively a change of cross-section from $R=0.05$ to $R=0.0316$ in the axisymmetric context). The initial conditions of the shock tube are summarised in table 21.

Table 21

Initial conditions of the shock tube for the test 1

\begin{tabular}{|c|c|c|c|c|c|}
\hline Position $(m)$ & $\phi$ & $\mathrm{R}(\mathrm{m})$ & $\rho\left(\mathrm{kg} . \mathrm{m}^{-3}\right)$ & $\mathrm{u}\left({\left.\mathrm{m} . \mathrm{s}^{-1}\right)}^{\mathrm{P}(\mathrm{Pa})}\right.$ \\
\hline \hline$x=[0: 0.7]$ & 1 & 0.05 & 35.6 & 0 & $30 \times 10^{5}$ \\
$x=[0.7: 1.4]$ & 1 & 0.05 & 1.1867 & 0 & $1 \times 10^{5}$ \\
$x=[1.4: 2]$ & 0.4 & 0.0316 & 1.1867 & 0 & $1 \times 10^{5}$ \\
\hline
\end{tabular}

- A regular cross-section reduction (test 2), i.e. corresponding to a linear transition from $\phi=1$ to $\phi=0.25$ (respectively a linear transition of the cross-section from $R=0.05$ to $R=0.025$ ). The initial conditions of the shock tube are summarised in table 22 .

Table 22

Initial conditions of the shock tube for the test 2

\begin{tabular}{|c|c|c|c|c|c|}
\hline Position $(m)$ & $\phi$ & $\mathrm{R}(\mathrm{m})$ & $\rho\left(k g . \mathrm{m}^{-3}\right)$ & $\mathrm{u}\left({\mathrm{m} . \mathrm{s}^{-1}}^{-}\right.$ & $\mathrm{P}(\mathrm{Pa})$ \\
\hline \hline$x=[0: 0.7]$ & 1 & 0.05 & 35.6 & 0 & $30 \times 10^{5}$ \\
$x=[0.7: 1.4]$ & 1 & 0.05 & 1.1867 & 0 & $1 \times 10^{5}$ \\
$x=[1.4: 1.7]$ & $1 \rightarrow 0.25$ & $0.05 \rightarrow 0.025$ & 1.1867 & 0 & $1 \times 10^{5}$ \\
$x=[1.7: 2]$ & 0.25 & 0.025 & 1.1867 & 0 & $1 \times 10^{5}$ \\
\hline
\end{tabular}

\subsection{The discontinuous transition case}

We first consider the situation of the abrupt transition. Table 21 gives the initial conditions of the shock tube while figures 52 show respectively the comparison between the one-dimensional model and the axisymmetric one (we display the cut at $r=0$ ) for the density and Mach number at two different times $t=1.3 \mathrm{~ms}$ (top) and $t=1.7 \mathrm{~ms}$ (bottom). At last, figure 53 gives the density isolines for the axisymmetric model at time $t=1.7 \mathrm{~ms}$. 

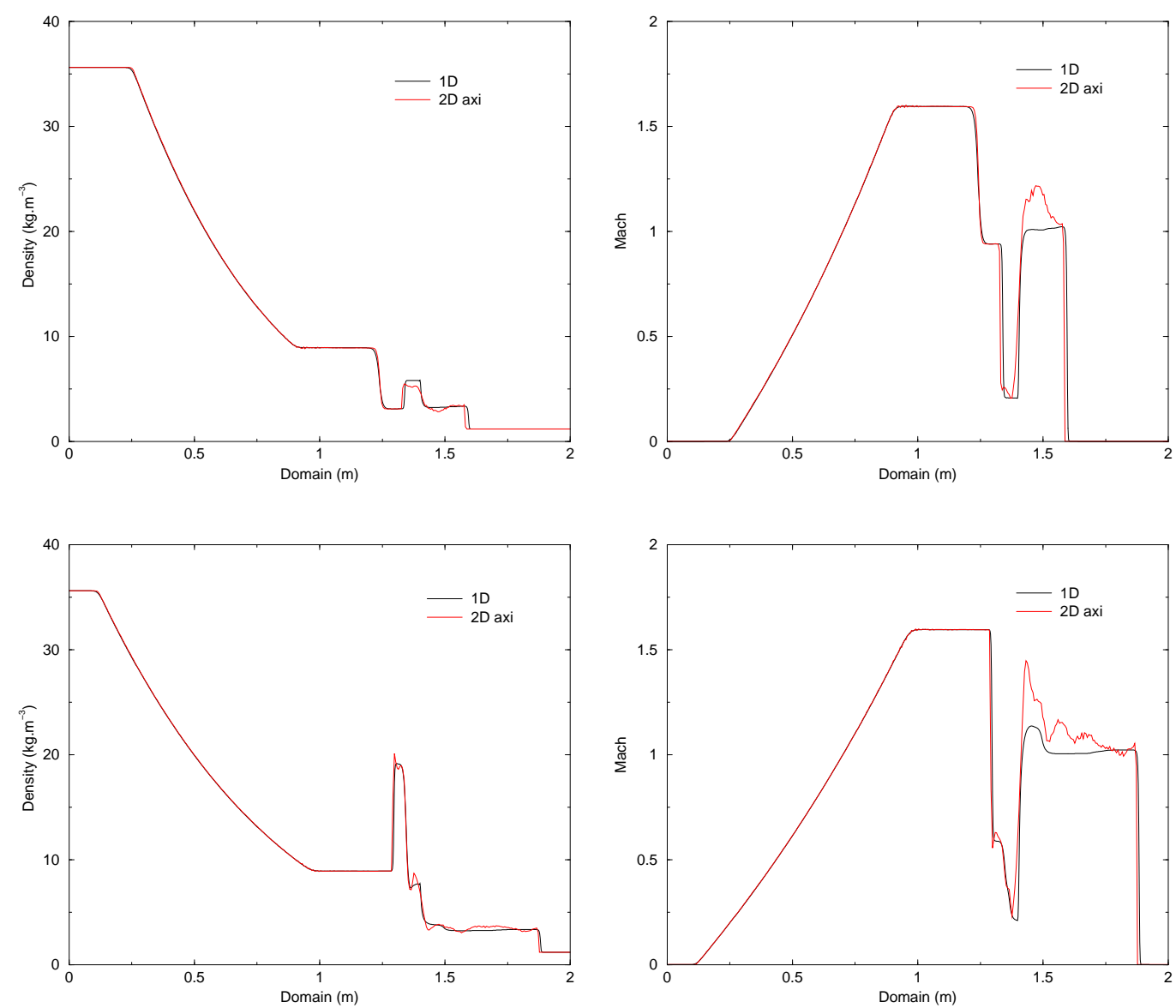

Fig. 52. Distribution of the density and the Mach number obtained by the two models at time $t=1.3 \mathrm{~ms}$ (top) and $t=1.7 \mathrm{~ms}$ (bottom) for the test 1 .

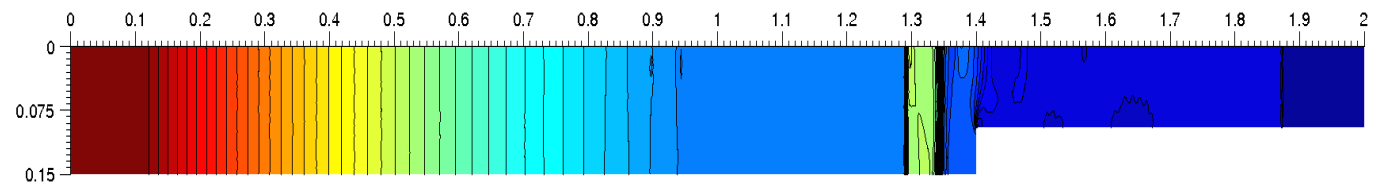

Fig. 53. 50 isodensity from 1 to 36 at time $t=1.7 \mathrm{~ms}$.

Comments. The incident shock wave generated by the Riemann problem is parted into a transmitted wave and a reflected wave at time $t=1.3 \mathrm{~ms}$ by the cross-section discontinuity located at $x=1.4 \mathrm{~m}$. Both the models give a similar behaviour but several differences can be highlighted. We first observe that the nonconservative model generates a higher reflected wave density and a lower transmitted wave density with respect to the axisymmetric model. The transition between the cross-section discontinuity and the transmitted shock (the $3-s$ shock) is also different: we note that the gas velocity increases just after the cross-section discontinuity for the axisymmetric model whereas the one-dimensional model presents a flat curve for the velocity. Nevertheless, we obtain a very good agreement between the two solutions, in particular the 
$3-s$ shock corresponding to the transmitted wave is the same in the two simulations.

\subsection{The smooth transition case}

We now deal with the smooth transition case where we use the same Riemann problem to generate the incident wave. Table 22 gives the initial conditions of the shock tube while figures 54 show respectively the comparison between the one-dimensional model and the axisymmetric one (we display the cut at $r=0$ ) for the density and Mach number at two different times $t=1.45 \mathrm{~ms}$ (top) and $t=1.76 \mathrm{~ms}$ (bottom). At last, figure 55 gives the density isolines for the axisymmetric model at time $t=1.76 \mathrm{~ms}$.
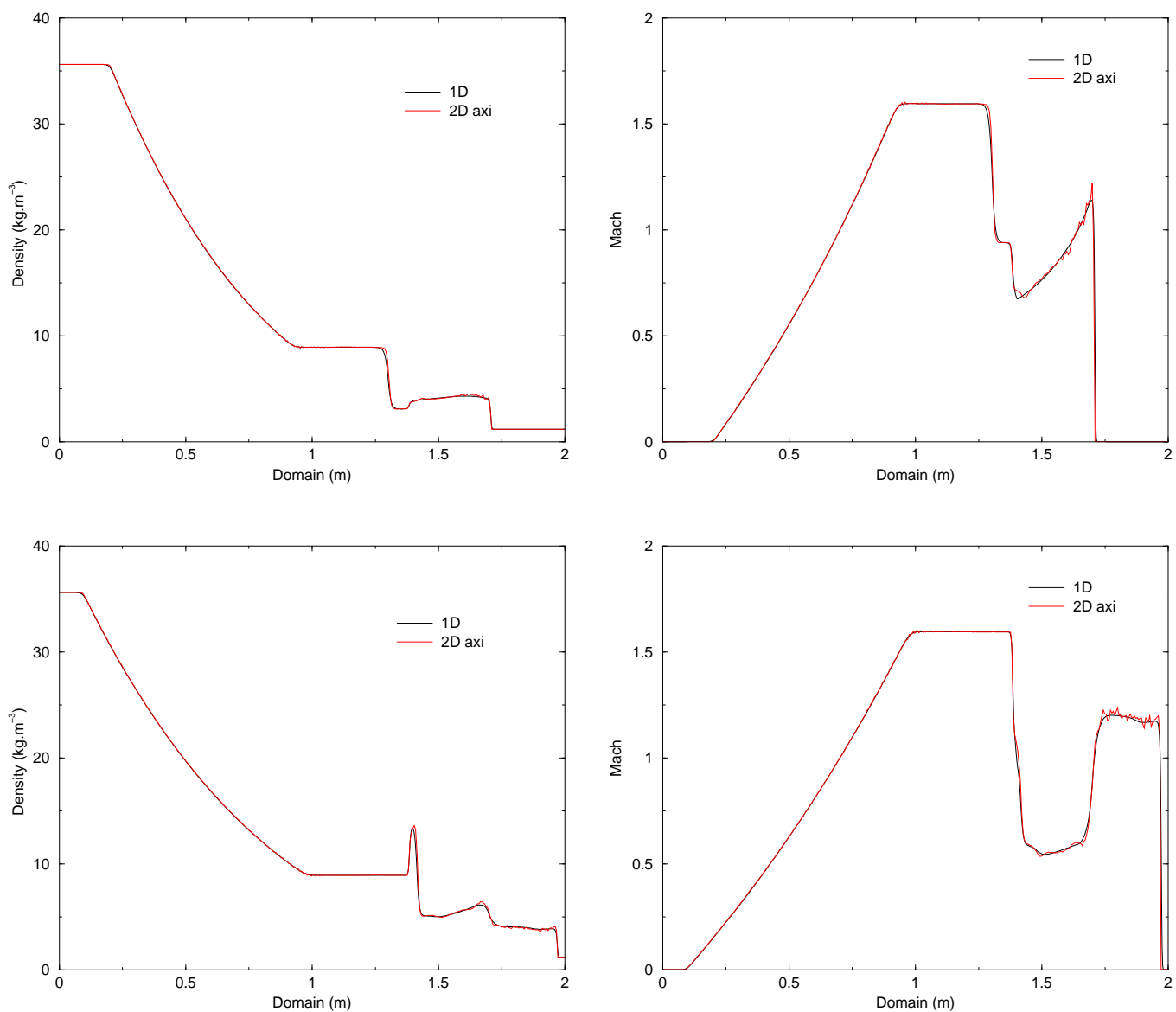

Fig. 54. Distribution of the density and the Mach number obtained by the two models at time $t=1.45 \mathrm{~ms}$ (top) and $t=1.76 \mathrm{~ms}$ (bottom) for the test 2 .

Comments. With a regular cross-section variation, we obtain a perfect agreement between the two models even in the delicate zone situated after the position $x=1.4 \mathrm{~m}$. Transitions between subsonic and supersonic states are also 


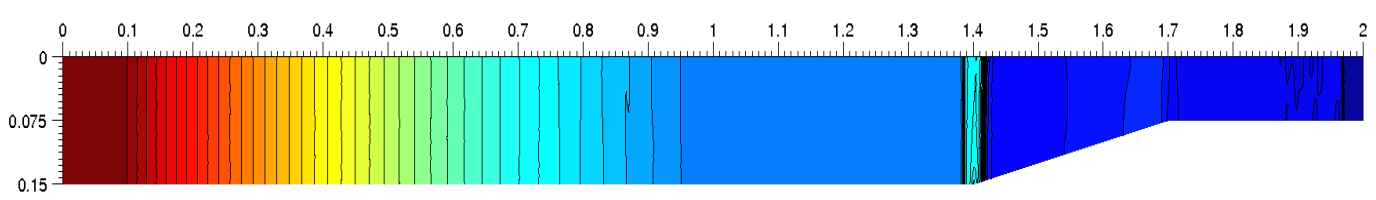

Fig. 55. 50 isodensity from 1 to 36 at time $t=1.76 \mathrm{~ms}$.

nice and, in this case, the one-dimensional nonconservative model is representative of the fully three-dimensional conservative one since there is no oblique wave generated by the cross-section variation. It is noticeable that the solution for regular variation situation is different to the abrupt one: the reflected and transmitted waves are smoothed and the intermediate states are different. For example, in the brutal variation case we observe a constant state just after the contact discontinuity $0-w$ whereas the density increases linearly in the smooth variation case due to the linear variation of the cross-section.

\section{Conclusion}

A systematic comparison of all the admissible configurations between the onedimensional nonconservative model and the axisymmetric conservative Euler system has been carried out. For the one-dimensional approach, we use the Rusanov flux adapted to the nonconservative Euler system proposed by [11] and the specific high-order scheme for the Euler system with cylindrical coordinates developed in [12]. Numerical results show a very good correspondence between the two models when the solutions of the axisymmetric model present straight longitudinal shocks i.e. no noticeable transversal shock perturbs the solution. Simulations based on the axisymmetric model also confirm the existence of complex configurations such as the $L R, R R, L R R$ and $R R R$ configurations introduced in [11].

We have tested the example of non uniqueness proposed by [3] and also proposed a new example of non uniqueness based on the $D$ and $R R R_{1}$ configurations. Indeed, two entropy solutions in the Lax sense are available and we show that the numerical scheme for the one-dimensional nonconservative problem does not always pick up the same solution obtained by the axisymmetric model.

In the last section, we have proposed a representative situation of engineering applications where a strong shock wave, generated upstream, comes to propagate in a converging duct. Numerical results have shown a good agreement between the two models even if the cross-section transition is abrupt.

\section{Acknowledgment}

This work was financially supported by the Schneider Electric company. 


\section{References}

[1] F. Alcrudo, F. Benkhaldoun Exact solutions to the Riemann problem of the shallow water equations with a bottom step Computers \& Fluids, Volume 30, Issue 6, July 2001, Pages 643-671.

[2] N. Andrianov, Performance of numerical methods on the non-unique solution to the Riemann problem for the shallow water equations, Int. J. Num. Meth. Fluids 47 (2005) 825-831.

[3] N. Andrianov and G. Warnecke, On the solution to the Riemann problem for the compressible duct flow, SIAM J. Appl. Math. 64(3) (2004) 878-901.

[4] N. Andrianov and G. Warnecke, The Riemann problem for the Baer-Nunziato two-phase flow model, J. Comput. Phys. 195 (2004), 434-464 .

[5] A. Britan, G. Ben-Dor, O. Igra and H. Shapiro, Shock waves attenuation by granular filters, International Journal of Multiphase Flow Volume 27 Issue 4 (2001) 617-634.

[6] C. Berthon, B. Nkonga, Multifluid numerical approximation based on a multipressure formulation, Computers and Fluids, 36 (2007) 467-479.

[7] T. Buffard, S. Clain, Monoslope and multislope MUSCL methods for unstructured meshes, Journal of Comptutational Physics, 229 (2010) 3745-3776.

[8] M. J. Castro Días, E. D. Fernández-Nieto, A. M. Ferreiro, Sediment transport models in shallow-water equations and numerical approach by high order finite volume methodes, Computers and Fluids, 37 (2008) 299-316.

[9] S. Clain, V. Clauzon, The multislope MUSCL method, Proceeding in the Finite Volumes for Complex Application 5, Wiley, (2008) 297-304.

[10] A. Chinnayya, A. Y. LeRoux and N. Seguin, A well-balanced numerical scheme for shallow-water equations with topography: resonance phenomena, International Journal on Finite Volumes (2004).

[11] S. Clain, D. Rochette First- and second-order finite volume methods for the onedimensional nonconservative Euler system, Journal of Computational Physics Volume 228 Issue 22 (2009) 8214-8248.

[12] S. Clain, D. Rochette, R. Touzani, A multislope MUSCL method on unstructured meshes applied to compressible Euler equations for swirling flows, Journal of Computational Physics, 229 (2010) 4884-4906.

[13] J.A. Désidéri, A. Goudjo, Un schéma de volumes-finis décentré pour la résolution des équations d'Euler en axisymétrique, INRIA Report No. 1005 (1989).

[14] M. Dumbser, M. Castro, C. Pares, E. F. Toro, ADER Schemes on Ustructured Meshes for Nonconservative Hyperbolic Systems: Applications to Geophysical Flows, Computers and Fluids, 38 (2009) 1731-1746. 
[15] E.D. Fernandez-Nieto, J. Marin, J. Monnier, Coupling superposed 1D and 2D shallow-water models: Source terms in finite volume schemes Computers \& Fluids, Volume 39, Issue 6, June 2010, Pages 1070-1082.

[16] P. Garcia-Navarro, M. E. Vazquez-Cendon, On numerical treatment of the source terms in the shallow water equations Computers \& Fluids, Volume 29, Issue 8, 31 August 2000, Pages 951-979.

[17] T. Gallouët, J.-M. Hérard, N. Seguin, Some approximate Godunov schemes to compute shallow-water equations with topography, Computers and Fluids, 32 (2003) 479-513.

[18] P. Glaister, Flux difference splitting for the Euler equations with axial symmetry, Journal of Engineering Mathematics, 22 (1988) 107-121.

[19] P. Goatin, P. G. Le Floch, The Riemann problem for a class of resonant hyperbolic systems of balance laws, Annales de l'Institut Henri Poincare (C) Non Linear Analysis Volume 21 Issue 6 (2004) 881-902.

[20] J. M. Greenberg and A. Y. Leroux, A well-balanced scheme for the numerical processing of source terms in hyperbolic equations, SIAM J. Numer. Anal. 33(1) (1996) $1-16$.

[21] A. Guardone, L. Vigevano, Finite element/volume solution to axisymmetric conservation laws, Journal of Computational Physics 224(2) (2007) 489-518.

[22] P.A. Jacob, Transient, hypervelocity flow in an axisymmetric nozzle, ICASE report No 91-1, NASA research center (1991).

[23] M.A. Leschziner, W. Rodi, Computation of strongly swirling axisymmetric free jets, AIAA Journal, 22 (1984) 1742-1747.

[24] C. Parés, Numerical methods for nonconservative hyperbolic systems: a theoritical framework, S.I.A.M. J. Numer. Anal., Vol 44, No 1 (2006) 300-321.

[25] C. Parés, M. Castro, On the well-balance property of Roe's method for nonconservative hyperbolic systems. Applications to shallow-water systems, ESAIM: mathematical modelling and numerical analysis, Vol. 38, No 5, 821852 (2004).

[26] D. Rochette, S. Clain, P. André, W. Bussière and F. Gentils, Two-dimensional modelling of internal arc effects in an enclosed MV cell provided with a protection porous filter, J. Phys. D: Appl. Phys. 40 (2007) 3137-3144.

[27] D. Rochette, S. Clain, F. Gentils, Numerical investigations on the pressure wave and the gas cooling interacting with a porous filter, during an internal arc fault in medium voltage cells, IEEE Transaction on Power Delivery, Vol. 23, No 1, 203-212 (Jan. 2008)

[28] G. Rosatti, L. Fraccarollo, A well-balanced approach for flows over mobile-bed with high sediment-transport, Journal of Computational Physics 220 (2006) 312-338.

[29] M. J. Zucrow, J. D. Hoffman, Gas dynamics, vol. 2, John Wiley \& Sons (1977). 